\title{
Comparison Between Parallel Hole and Rotating Slat Collimation: Analytical Noise Propagation Models
}

\author{
Lin Zhou, Michel Defrise, Kathleen Vunckx and Johan Nuyts
}

\begin{abstract}
We have previously proposed a method to compare tomographic systems. It is assumed that each system acquires a tomographic scan of a certain tracer distribution in the same acquisition time. From this scan, each system is forced to reconstruct an image with a predefined spatial resolution. The system that can perform this task with the "most favorable" noise propagation is considered as the best system. The variance on pixel values or region-of-interest (ROI) values is used to assess the noise in the reconstructed image. In this paper, we extend this idea to compare the performance of parallel hole $(\mathrm{PH})$ and rotating slat (RS) collimations.

Two different analytical approaches were used to analyze the variance of the reconstructed pixel/ROI values. The first method is based on the filtered-backprojection (FBP) theory, and was applied to the central point of a uniform symmetrical phantom. It yields analytical expressions for the optimal collimator aperture and the corresponding variance of the reconstructed pixel values, but it can only be applied to highly symmetrical configurations. The second method is based on approximations for the Fisher information matrix. It provides numerical results, and it is more general and can be applied to non-symmetrical objects and shiftvariant tomographic systems.

The collimations were compared for both planar imaging and volume imaging. The main results are: 1) For cases where both methods are valid, they are in excellent agreement. 2a) The optimal collimator aperture varies linearly with the target resolution. $2 \mathrm{~b}$ ) For a fixed target resolution, the optimal collimator aperture depends on the collimator type and the imaging mode (planar or volume). 2c) The optimal aperture of $\mathbf{P H}$ is a factor of $\sqrt{2}$ larger than that of RS. 3a) The relative performance of the two collimators is determined by both the object size and the objectto-detector distance. 3b) Pixel variance and variances of ROIs with varying sizes yield very similar relative performance for $\mathrm{RS}$ versus PH.
\end{abstract}

\section{INTRODUCTION}

In single photon emission computed tomography (SPECT) imaging, image quality is limited by the tradeoff between the spatial resolution and the geometric efficiency. For the conventional parallel hole (PH) collimator (Fig. 1(a)), the geometric efficiency is always very low for a reasonable spatial resolution since PH only detects photons whose trajectories are parallel to the collimator holes. In contrast to $\mathrm{PH}$, a rotating slat (RS) collimator (Fig. 1(b)) can achieve a much higher geometric

L.Zhou, K.Vunckx and J.Nuyts are with the Dept. of Nuclear Medicine, K.U.Leuven, B-3000 Leuven, Belgium. M.Defrise is with the Dept. of Nuclear Medicine, V.U.Brussel, B-1090 Brussel, Belgium

This work is supported by F.W.O. grant G.0569.08. and I.U.A.P. grant NIMI Copyright (c) 2010 IEEE. Personal use of this material is permitted. However, permission to use this material for any other purposes must be obtained from the IEEE by sending a request to pubs-permissions@ieee.org.

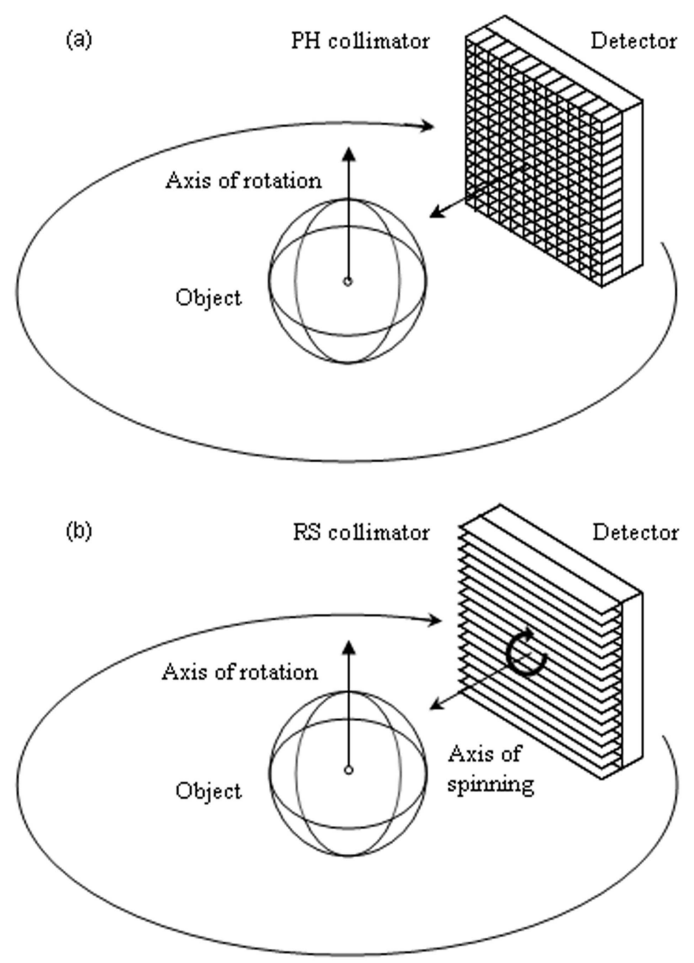

Fig. 1. (a) Geometry of a parallel hole (PH) collimator system. (b) Geometry of a rotating slat (RS) collimator system.

efficiency without any resolution loss due to its capability of in-plane photons collection [1]-[10]. However, in order to interpret the plane integral data acquired by RS, even for planar imaging, an extra reconstruction step is needed, which results in increased noise propagation. It is not obvious whether or not a higher geometric efficiency will lead to improved image quality. The goal of our study is to compare the $\mathrm{PH}$ and the RS collimators with different analytical noise propagation models, investigating which collimator provides better image quality in the reconstructions.

To compare different tomographic systems, it is important to state clearly which figure of merit (FOM) will be used and for which kind of imaging task the comparison will be performed. Indeed, there are various FOMs and tasks that can be applied to the system comparison, e.g., the signal-to-noise ratio (SNR) of a human observer for a lesion detection task, or the mean and the variance of an estimator in a estimation task [11]. In our previous studies, we used the estimate of the variance of the 
reconstructed pixel or region-of-interest (ROI) value to evaluate the noise propagation characteristics of different tomographic systems. For fair comparison, we assume that each system acquires a tomographic scan of the same tracer distribution in the same acquisition time and we tune the reconstruction algorithm for each system in such a way that all reconstructed images have the same predefined spatial resolution. The system that yields the lowest variance can then be considered as the best system. This approach was used in [12] for the selection of the best design among various multi-pinhole configurations. In this paper, we extend this idea to compare the $\mathrm{PH}$ and the RS collimators.

Comparative studies between these two collimator systems have already been presented in several papers [5]-[10]. In [5] and [6], a specific expression of the SNR gain (RS versus $\mathrm{PH})$ at the center of a uniform disk/sphere of activity was derived for planar and volume imaging, respectively. The comparison was based on the noise propagation behavior of filtered-backprojection (FBP) reconstruction. It was found that RS outperformed PH only for small objects. Effort has been made in [6] to achieve an isotropic point spread function for each collimator, but the final spatial resolutions yielded by the two systems were not really identical. Zhang and Zeng [7] compare these two collimators using the same figure of merit and the same type of phantom as in [6] for the iterative least squares (LS) reconstruction, yielding similar results as [6]. In that study, the variation of the geometric efficiency within the field of view (FOV) and the distance-dependent detector response were not taken into consideration. In [8], [9], the $\mathrm{PH}$ and the RS systems were compared using maximum likelihood expectation maximization (MLEM) reconstruction with the system model derived from Monte Carlo simulations. The contrast-to-noise for cold and hot spots with varying sizes was investigated at different iterations. It was found that the PH collimator only outperforms RS for low contrast lesions in very large warm background. The results are reliable because they were derived based on accurate system models. However, the analysis was done with fixed object-to-detector distances. Since the geometric efficiency of the RS collimator is positiondependent while that of $\mathrm{PH}$ is constant, changing the detector distance may have a significant influence on the outcome of the collimator comparison. Wang et al [10] performed a real lesion phantom study. The data were reconstructed using the rescaled block-iterative expectation maximization (RBI-EM) algorithm. The contrast-to-noise analysis (similar as in [8], [9]) showed that RS leads to a better contrast recovery than $\mathrm{PH}$ for the same background noise level. Since different detectors were used for the two collimator systems ( $\mathrm{NaI}$ for $\mathrm{PH}$ and $\mathrm{CdZnTe}$ (CZT) for RS), it was not clear how much of the improvement was due to the high geometric efficiency of RS and how much was due to the better energy resolution of the CZT detector.

Besides the points discussed above, the tradeoff between the collimator resolution and the noise has not yet been fully explored in previous works. For the same predefined spatial resolution (called target resolution in the remainder of the text), the variance of the reconstructed image varies with the collimator resolution, in a way that is specific to each type of collimator. The main innovation in the present paper is the investigation of the relation between the variance and the collimator resolution for the $\mathrm{PH}$ and RS collimators and the comparison of these two types of systems using for each the collimator resolution that yields the minimal pixel/ROI variance at fixed target resolution. To avoid the confusion between the resolution in the reconstruction and the collimator resolution, we introduce the term of collimator aperture to denote the collimator resolution. The collimator aperture which corresponds to the minimal variance is thus defined as the optimal collimator aperture of a certain collimator system.

The purpose of this study is therefore to make a fair comparison between the $\mathrm{PH}$ and the RS systems. The word "fair" in this context refers to the following:

- The two systems are equipped with the same type of detector with the same detection area,

- the object-to-detector distance is the same,

- the acquisition is done for the same tracer distribution in the same amount of time,

- the reconstructions have the same spatial target resolution,

- and as mentioned above we use the collimator aperture that is optimized individually for each system.

We used two analytical approaches in this study. The first one is based on the theory of FBP reconstruction and is similar to the work in [5], [6]. The FBP-based method uses a simplified system model and closed form expressions can only be obtained for highly symmetrical configurations. The second approach is the Fisher information-based method (FIM), which gives fast predictions of the linearized local impulse response and of the covariance image [13]-[17] for iterative reconstructions. In this study, the FIM was used to estimate the noise properties of the post-smoothed MLEM reconstruction. Compared with the FBPbased method, one of the advantages of FIM is that it allows the implementation of more accurate physical models. Practical considerations, such as the attenuation within the phantom, the position dependence of the geometric efficiency as well as the effect of the collimator blurring, can be conveniently included in the system model. Therefore FIM was used to generalize and extend the results yielded by the FBP-based calculation.

We applied these two methods to uniform symmetrical phantoms and investigated for each type of collimator the relation between the optimal collimator aperture and the target resolution. With the optimal collimator apertures corresponding to a certain target resolution, the variance of the reconstructed pixel/ROI values obtained with the two systems were compared as a function of either the phantom size or the object-to-detector distance. The study was done for both planar imaging and volume imaging and the results were verified with simulations.

This paper is organized as follows. Section II-A describes the geometry of the $\mathrm{PH}$ and the RS systems, the concept of the collimator aperture and the geometric efficiency. Section II-B gives general descriptions about the data acquisition and the reconstruction with the target resolution. Section II-C 
introduces the figure of merit used for system comparison. Section II-D and II-E describe the two proposed methods (FBP and FIM). Section II-F explains how to optimize the collimator aperture. Details about the numerical experiments are given in Section III. The results obtained from the two methods are presented in Section IV and V, respectively. The results are further discussed in Section VI and our conclusions are given in Section VII. Details of all the mathematical deviations are presented in Appendices.

\section{Methods}

\section{A. System Description}

1) Collimator Geometry: We consider a $\mathrm{PH}$ collimator consisting of a two-dimensional (2D) array of long narrow square holes with length $d_{p}$ and height $h_{p}$ (Fig. 1(a)). The $\mathrm{RS}$ collimator is modeled as a series of long thin slat septa positioned parallel with each other and perpendicular to the detector surface (Fig. 1(b)). The slat septa have a width $W$ and a height $h_{r}$. The distance between two adjacent slat septa is $d_{r}$. Both the $\mathrm{PH}$ and the $\mathrm{RS}$ collimators were equipped with a square detector array with size $W \times W$. In this study we assume that there is no penetration through the septa, that the thickness of the septa is negligible, and that the detectors have a perfect absorption efficiency and a perfect intrinsic detector resolution. The distance $D$ between the center of the image space and the detector surface is constant during the tomographic acquisitions.

For the RS system, the collimator and the detector need to spin around a spinning axis in order to acquire complete data. This axis is perpendicular to the detector surface, connecting the center of the detector array and the center of the image space. For tomographic scans, both the $\mathrm{PH}$ and the RS collimators rotate around the object. The axis of rotation (AOR) is the line parallel to the detector surface through the center of the image space.

2) Collimator Aperture: The collimator aperture (which denotes the collimator resolution) is a measure of the response of a collimator system to a point source, characterized by the point spread function (PSF). The $\mathrm{PSF}^{1}$ is here denoted as $P_{c}$ and is modeled by a Gaussian with standard deviation of $\sigma_{c}$, where the subscript $c$ represents "collimator" and can be substituted by either $p$ or $r$ to refer to the PH or the RS collimator, respectively (the same convention will be used for other quantities below). The collimator aperture can be expressed by either the full width at half maximum $\left(\mathrm{FWHM}_{c}\right)$ of the PSF or by its standard deviation, which for a Gaussian PSF is given by

$$
\sigma_{c}=\frac{\mathrm{FWHM}_{c}}{2 \sqrt{2 \ln 2}}
$$

Generally speaking, the collimator response is depth-dependent. For the proposed collimator geometries we have at a location $\vec{x}$ in the image space, [18]

$$
\operatorname{FWHM}_{c}(\vec{x})=D(\vec{x}) d_{c} / h_{c}
$$

${ }^{1}$ For the RS system, it only refers to the PSF profile along the dimension perpendicular to the collimator septa. where $D(\vec{x})$ is the distance between $\vec{x}$ and the detector plane. Combining Eq. (1) and Eq. (2) yields

$$
\sigma_{c}(\vec{x})=\frac{D(\vec{x}) d_{c}}{2 \sqrt{2 \ln 2} h_{c}}
$$

We use the symbol $\sigma_{c}$ or $\mathrm{FWHM}_{c}$ (without argument $\vec{x}$ ) to denote the collimator aperture that corresponds to the center of the image space, i.e.

$$
\sigma_{c}=\frac{D d_{c}}{2 \sqrt{2 \ln 2} h_{c}}
$$

3) Geometric Efficiency: The geometric efficiency of a collimation system $\left(E_{c}\right)$ is the fraction of the photons emitted from a point in the object that will be detected if there is no attenuation. The geometric efficiency is closely related to the collimator aperture. Generally, $E_{p}$ is proportional to $\sigma_{p}^{2}$, and $E_{r}$ is proportional to $\sigma_{r}$. In addition, $E_{r}$ also depends on the detector width $W$, because a wider detector spans a larger solid angle. However, the detection efficiency decreases with increasing incidence angle, and therefore we introduce an effective detector width $\Omega_{W}$ which takes a normalized angular factor into account. Appendix-A gives the specific definition of $\Omega_{W}$. With this definition, $E_{r}$ is proportional to $\Omega_{W}$, which leads to concise expressions.

Due to the collimator geometry, $E_{r}$ is position-dependent while $E_{p}$ is not. Expressions of $E_{p}$ and $E_{r}$ can be found in Appendix-B.

\section{B. Acquisition Model and Reconstruction}

1) Acquisition Model: The tracer distribution is denoted as $\lambda(\vec{x})$, with $\vec{x}$ the vector in the image space. It is nonnegative, and $\lambda(\vec{x})=0$ for $\vec{x} \notin S$, with $S$ the finite support of $\lambda(\vec{x})$.

For planar imaging, $\lambda(\vec{x})$ represents a $2 \mathrm{D}$ distribution $(\vec{x} \in$ $\mathbb{R}^{2}$ ) in a plane parallel to the detector surface. During the acquisition, the $\mathrm{PH}$ collimator is stationary and the RS collimator spins around the spinning axis. The acquired data for the $\mathrm{PH}$ system is here modeled as

$$
y_{p}(\vec{x})=T_{p} E_{p} \int_{\mathbb{R}^{2}} P_{p}(\|\vec{x}-\vec{\xi}\|) \lambda(\vec{\xi}) d \vec{\xi}
$$

where $T_{p}$ is the acquisition time, $E_{p}$ is the geometric efficiency, $P_{p}$ is the PSF which models the collimator blurring, and $\|\cdot\|$ represents the Euclidean norm. As discussed above, $P_{p}$ is a 2D isotropic Gaussian with standard deviation of $\sigma_{p}$.

The planar RS acquisition is modeled as

$y_{r}(s, \phi)=T_{r} \int_{-W / 2}^{+W / 2} d l \int_{\mathbb{R}^{2}} E_{r}(\alpha-l, D) P_{r}(|s-\beta|) \lambda(\vec{\xi}) d \alpha d \beta$

with

$$
\vec{\xi}=\alpha \vec{u}_{\phi}^{\perp}+\beta \vec{u}_{\phi}
$$

where $s$ is the distance between the slat interval and the spinning axis, $\phi$ is the spinning angle, $\vec{u}_{\phi}$ and $\vec{u}_{\phi}^{\perp}$ are two orthogonal unit vectors in the detector plane, with $\vec{u}_{\phi}=$ $(-\sin \phi, \cos \phi)$ orthogonal to the slat septa. Note that for this 
2D imaging configuration, the distance $D$ between the source and the detector plane is fixed and the geometric efficiency $E_{r}$ (see Eq. (B-2) in Appendix) only varies along $\vec{u}_{\phi}^{\perp} . P_{r}$ is the 1D PSF spread along $\vec{u}_{\phi}$. Assuming equal acquisition time for both collimator systems, we have

$$
T_{r}=T_{p} / \pi
$$

because RS spins over $\pi$ during the acquisition.

For volume imaging, $\lambda(\vec{x})$ represents a 3D distribution $\left(\vec{x} \in \mathbb{R}^{3}\right)$. For both collimator systems, the collimator and the detector rotate around the AOR. The acquired data of the PH system is modeled as

$$
\begin{aligned}
y_{p}(v, \theta, z) & =T_{p} E_{p} \int_{\mathbb{R}^{3}} P_{p}\left(\sqrt{(v-\alpha)^{2}+(z-\beta)^{2}}, \gamma\right) \\
& \times \operatorname{Atten}(\vec{\xi}) \lambda(\vec{\xi}) d \alpha d \beta d \gamma
\end{aligned}
$$

with

$$
\vec{\xi}=\alpha \vec{u}_{v}+\beta \vec{u}_{z}+\gamma \vec{u}_{\theta}
$$

where $\theta$ is the acquisition angle with respect to the AOR, and $v$ and $z$ are the 2D orthogonal coordinates in the detector array, defined by unit vectors $\vec{u}_{v}, \vec{u}_{z}$, respectively, with $\vec{u}_{z}$ parallel to the AOR. The third unit vector $\vec{u}_{\theta}=(-\sin \theta, \cos \theta, 0)$ is orthogonal to the detector plane. $\operatorname{Atten}(\vec{\xi})$ represents the probability that a photon, emitted from $\vec{\xi}$ and traveling along $\vec{u}_{\theta}$, is not attenuated.

The data acquired by the tomographic RS system is modeled as

$$
\begin{aligned}
y_{r}(s, \phi, \theta) & =T_{r} \int_{-W / 2}^{+W / 2} d l \int_{\mathbb{R}^{3}} E_{r}(\alpha-l, \gamma) P_{r}(|s-\beta|, \gamma) \\
& \times \operatorname{Atten}(\vec{\xi}, l) \lambda(\vec{\xi}) d \alpha d \beta d \gamma
\end{aligned}
$$

where

$$
\vec{\xi}=\alpha \vec{u}_{\phi}^{\perp}+\beta \vec{u}_{\phi}+\gamma \vec{u}_{\theta}
$$

with $\vec{u}_{\phi}^{\perp}=(\cos \phi \cos \theta, \cos \phi \sin \theta, \sin \phi)$ and $\vec{u}_{\phi}=$ $(-\sin \phi \cos \theta,-\sin \phi \sin \theta, \cos \phi)$. In Eq. (11), $\operatorname{Atten}(\vec{\xi}, l)$ has a similar meaning as $\operatorname{Atten}(\vec{\xi})$ in Eq. (9), with trajectory of the photon from $\vec{\xi}$ to the detector position $l$.

Note that in Eqs. (9) and (11), the PSF $P_{c}(\cdot, \gamma)$ models a $\operatorname{depth}(\gamma)$-dependent collimator response, representing a Gaussian with standard deviation of $\sigma_{c}(\gamma)$ given by Eq. (3).

The decay of the tracer during the acquisition and the photon scattering are not included in our model, and the data is assumed to be Poisson distributed.

2) Reconstruction with Target Resolution: From $y_{p}$ and $y_{r}$ we reconstruct the image using either FBP or MLEM, with a spatial resolution equal to the predefined target resolution. The reconstruction is achieved by making an initial (nearly) unbiased reconstruction, which is then smoothed with a postprocessing filter to impose the target PSF $\left(P_{t}\right)$, in our case an isotropic Gaussian with target standard deviation $\sigma_{t}$.

To ensure that the reconstruction is nearly unbiased, the blurring due to the finite collimator aperture needs to be compensated during the reconstruction. With FBP, this is done by deconvolving the acquired data for the collimator PSF. With MLEM, the effect of the collimator blurring is included in the system model. It was assumed that a nearly unbiased reconstruction is achieved by iterating MLEM until convergence.

To estimate the resolution and the noise properties in the reconstruction, we followed the approach of [13]-[17]. We consider a discrete model of the acquisition, i.e., both the image space and the detection space are digitized into pixels with finite size. Let $\Lambda=\left[\lambda_{1}, \lambda_{2}, \cdots, \lambda_{N}\right]^{T}$ be the true tracer distribution, where $N$ is the number of pixels in the image space and ${ }^{T}$ denotes the transpose. Let $Y=\left[y_{1}, y_{2}, \cdots, y_{M}\right]^{T}$ be the expectation of the acquired data (actual measured data will deviate from $Y$ because of the noise), with $M$ the number of the projection elements. $\hat{\Lambda}(Y(\Lambda))$ is the expectation of the reconstruction from $Y(\Lambda)$. We use the linearized local impulse response (LLIR) at a particular pixel $j$ in $\hat{\Lambda}$ to describe the resolution property. This LLIR is defined as

$$
l^{j}(\hat{\Lambda})=\lim _{\delta \rightarrow 0} \frac{\hat{\Lambda}\left(Y\left(\Lambda+\delta e^{j}\right)\right)-\hat{\Lambda}(Y(\Lambda))}{\delta}
$$

where $e^{j}$ is the $j$-th unit vector with dimension of $N \times 1$. The vector $l^{j}$ contains the response of the reconstructed image to a tiny disturbance $\delta$ at $j$ in the true tracer distribution. This definition enables the application of LLIR to non-linear and/or shift variant systems, where the conventional definition of $\mathrm{PSF}^{2}$ cannot be used. For linear shift invariant systems, LLIR and PSF are identical.

The local impulse response contrast recovery coefficient (CRC) is defined as the value of $l^{j}$ in pixel $j$

$$
\mathrm{CRC}^{j}=l^{j}[j]
$$

By definition, a larger CRC indicates a sharper LLIR, meaning that a better resolution is achieved in the reconstruction. Therefore we use here the CRC as a measure of resolution.

We denote the variance of the reconstructed value of pixel $j$ as $\operatorname{VAR}^{j}$. This is the expectation of the variance due to noise propagation, which can be estimated from repeated measurements. The contrast-to-noise ratio (CNR) in $j$ is computed as

$$
\mathrm{CNR}^{j}=\mathrm{CRC}^{j} / \sqrt{\mathrm{VAR}^{j}}
$$

We use CNR as the figure of merit for the system comparison.

In this study, we choose $\sigma_{t}>\sigma_{c}$, such that post-smoothing is always needed to achieve the target resolution. The postsmoothing filter is designed based on the deviation between the LLIR $l^{j}$ of the nearly-unbiased initial reconstruction and the target PSF $P_{t}$. Applying this filter to the nearly-unbiased initial reconstruction then ensures that the final $l^{j}$ equals $P_{t}$. By this means, $\mathrm{CRC}^{j}$ is constant for the given target resolution and $\mathrm{CNR}^{j}$ depends only on $\mathrm{VAR}^{j}$.

The discrete model described in this section is directly applied in the Fisher information-based method, which predicts

\footnotetext{
${ }^{2}$ The PSF mentioned here is different from the PSF $P_{c}$ introduced in IIA. This PSF is in the reconstructed image space and is influenced by both the system geometry and the reconstruction algorithm. It is applied to shiftinvariant system to describe the global resolution property. The PSF $P_{c}$ is in detection space and is only influenced by the collimator geometry.
} 
the noise properties of the post-smoothed MLEM reconstruction. As for the FBP-based method, we use the continuous data acquisition model and the variance is calculated for a point rather than for a pixel with finite size. The difference is only apparent however because the variance is calculated in both cases for a smoothed image with a prescribed target resolution $\sigma_{t}$. As long as the pixel size is sufficiently small compared to the target resolution (which is the case in our study), the effect of the discretization is small and can be considered negligible. The calculated variance actually corresponds to the detection of a (non-uniform) ROI with a Gaussian profile with standard deviation $\sigma_{t}$.

Details about the two methods are presented in II-D and II-E, respectively.

\section{Performance Measure}

We propose to measure the relative performance of the two systems using the ratio (gain) between the $\mathrm{CNR}^{j}$ obtained with the RS and PH collimators. For convenience, the superscript ${ }^{j}$ is dropped in the equation

$$
\text { Gain }_{\mathrm{CNR}}=\frac{\mathrm{CNR}_{r}}{\mathrm{CNR}_{p}}=\frac{\mathrm{CRC} / \sqrt{\mathrm{VAR}_{r}}}{\mathrm{CRC} / \sqrt{\mathrm{VAR}_{p}}}=\sqrt{\frac{\mathrm{VAR}_{p}}{\mathrm{VAR}_{r}}}
$$

Note that CRC is canceled out because it is the same for both collimator systems as we impose equal target resolution.

Eq. (16) is computed using the two proposed methods presented below. With the second method, the CNR gains for ROIs of varying sizes are also investigated, with more details presented in II-E.

\section{FBP-Based Calculation}

The comparative study between the $\mathrm{PH}$ and the RS collimator systems is first performed based on the theory of FBP reconstruction.

The object $\lambda(\vec{x})$ is a uniform disk or a uniform sphere for planar and volume imaging, respectively. The center of $\lambda(\vec{x})$ coincides with the center of the image space. To simplify the calculation, the following approximations are made in Eqs. (5), (6), (9) and (11): 1) There is no attenuation within the object, i.e. $\operatorname{Atten}(\vec{\xi})=1$, and 2) The position-dependence of the geometric efficiency and of the collimator response is ignored, i.e., $\gamma$ is replaced by $D$ (distance between the center of the image space and the detector) in the second argument of $E_{r}, P_{p}$, and $P_{r}$. Thus, the effect of the collimator smoothing is modeled by a single convolution between the data and a Gaussian with standard deviation $\sigma_{c}$. In addition, when calculating the variance at the center of the object, the variance of the data acquired at the center of the detector is used to approximate the variance of the data of all the other detectors.

To reconstruct the data, the conventional way is to apply a ramp filter (for 2D RS or 3D PH imaging) or a second derivative filter [19] (for 3D RS imaging) to the acquired data, and then perform back-projection. To impose the target resolution $\sigma_{t}$, an extra filter which corresponds to a Gaussian kernel with a standard deviation of $\sqrt{\sigma_{t}^{2}-\sigma_{c}^{2}}$ is applied together with the reconstruction filters before the back-projection, which is equivalent to post-smooth the initial unbiased reconstruction.

Based on the reconstruction, the variance of the central point of the reconstruction is derived as a function of the collimator aperture $\sigma_{p}(\mathrm{PH})$ or $\sigma_{r}(\mathrm{RS})$. This variance is further minimized by choosing an optimal value for the collimator aperture (See II-F). The minimal variance for each collimator system is used in Eq. (16) to evaluate of the relative performance of the two collimator systems.

This method is only valid for the central point of a uniform symmetrical object. Detailed calculations can be found in Appendix-C and Appendix-D.

\section{E. Fisher Information-Based Method}

The second approach is the Fisher information-based method. FIM was first proposed in [13]-[15] for converged maximuma-posteriori (MAP) reconstruction. Later it was adapted and validated for the post-smoothed MLEM reconstruction with a target resolution [16], [17]. In this study, we use FIM to predict the LLIR and the covariance matrix, which describes the noise correlation between the pixels of the post-smoothed MLEM reconstruction.

1) Matrix Description: FIM assumes discrete data and is most conveniently presented in matrix notation. As before, the activity distribution is $\Lambda=\left[\lambda_{1}, \lambda_{2}, \cdots, \lambda_{N}\right]^{T}$ and the acquired data is $Y=\left[y_{1}, y_{2}, \cdots, y_{M}\right]^{T}$. The data is expressed as

$$
Y=A \Lambda
$$

where the $M \times N$ matrix $A$ is the system matrix whose element $a_{i j}$ represents the probability that photons emitted from pixel $j$ will be detected in detector unit $i$. The reconstruction from the data $Y$ is denoted as $\hat{\Lambda}$.

Eq. (17) is implemented by discretizing Eqs. (5)-(12), with $A$ including the combined effect of the position-dependent geometric efficiency, the depth-dependent collimator response, as well as the attenuation within the object.

2) Basic Equations: For a pixel of interest $j$ in $\hat{\Lambda}$, the LLIR $\left(l^{j}\right)$ and the corresponding covariance matrix $\left(\mathrm{Cov}^{j}\right)$ are approximated as [16]

$$
\begin{aligned}
l^{j}(\hat{\Lambda}) & \approx \boldsymbol{P} \boldsymbol{G F} e^{j} \\
\operatorname{Cov}^{j}(\hat{\Lambda}) & \approx \boldsymbol{P} \boldsymbol{G F} \boldsymbol{G}^{T} \boldsymbol{P}^{T} e^{j}
\end{aligned}
$$

with

$$
\boldsymbol{F}=A^{T} C_{Y}^{-1} A
$$

where $\boldsymbol{F}$ is the Fisher information matrix, which is calculated by a forward projection $A$, weighted by the inverse of the covariance matrix $C_{Y}$ of the acquired data $Y$, and followed by a back-projection $A^{T}$. $\boldsymbol{G}$ is the approximated pseudoinverse of $\boldsymbol{F}$ [16]. $\boldsymbol{P}$ is the post-processing filter that imposes the prescribed target resolution. $\boldsymbol{F}, \boldsymbol{G}$ and $\boldsymbol{P}$ are all $N \times N$ matrices.

Since we are only interested in local properties of pixel $j$, it is reasonable to assume that locally $\hat{\Lambda}$ can be considered as shift-invariant [14]. With this approximation, $\boldsymbol{F}$ can be turned 
into a circulant matrix $\boldsymbol{F}^{j}$ where each row is a one element right-shifted version of the preceding row and the $j$-th row coincides with the $j$-th row of $\boldsymbol{F}$. Accordingly, $\boldsymbol{G}$ and $\boldsymbol{P}$ also become $j$-dependent, and Eqs. (18) and (19) are turned into

$$
\begin{aligned}
l^{j}(\hat{\Lambda}) & \approx \boldsymbol{P}^{j} \boldsymbol{G}^{j} \boldsymbol{F}^{j} e^{j} \\
\operatorname{Cov}^{j}(\hat{\Lambda}) & \approx \boldsymbol{P}^{j} \boldsymbol{G}^{j} \boldsymbol{F}^{j} \boldsymbol{G}^{j T} \boldsymbol{P}^{j T} e^{j}
\end{aligned}
$$

The advantage of Eqs. (21) and (22) is that they can be efficiently calculated in the Fourier domain [15], [16].

The post-smoothing filter $\boldsymbol{P}^{j}$ is defined in the following way

$$
\mathcal{P}[k]= \begin{cases}\mathcal{G}[k] / \mathcal{L}_{0}[k] & \text { if } \mathfrak{R}\left(\mathcal{L}_{0}[k]\right)>0.005, \\ 0 & \text { if } \mathfrak{R}\left(\mathcal{L}_{0}[k]\right)<0.005\end{cases}
$$

where $\mathcal{P}, \mathcal{G}, \mathcal{L}_{0}$ are the Fourier transforms of $\boldsymbol{P}^{j}, P_{t}$ and $l_{0}^{j}$, with $l_{0}^{j}=\boldsymbol{G}^{j} \boldsymbol{F}^{j} e^{j}$ the LLIR before post-smoothing, $k$ is the index of the elements in the Fourier domain, and $\mathfrak{R}(\cdot)$ denotes the real part of a complex number.

By definition, the CRC and the variance are the $j$-th elements of $l^{j}(\hat{\Lambda})$ and $\operatorname{Cov}^{j}(\hat{\Lambda})$, respectively. The CNR thus becomes

$$
\begin{aligned}
\mathrm{CNR}^{j} & =\mathrm{CRC}^{j} / \sqrt{\operatorname{VAR}^{j}} \\
& \simeq e^{j T} \boldsymbol{Q}_{L}^{j} e^{j} / \sqrt{e^{j T} \boldsymbol{Q}_{C}^{j} e^{j}}
\end{aligned}
$$

with $\boldsymbol{Q}_{L}^{j}=\boldsymbol{P}^{j} \boldsymbol{G}^{j} \boldsymbol{F}^{j}$ and $\boldsymbol{Q}_{C}^{j}=\boldsymbol{P}^{j} \boldsymbol{G}^{j} \boldsymbol{F}^{j} \boldsymbol{G}^{j T} \boldsymbol{P}^{j T}$.

As discussed in II-B.2, CRC $^{j}$ is constant for a given target resolution. The (co)variance in the reconstruction is proportional to the measured activity $Y$ (with $\mathrm{Cov}^{j} \propto \boldsymbol{F}^{-1}, \boldsymbol{F} \propto C_{Y}^{-1}$ and $C_{Y} \propto Y$ ), and inversely proportional to the acquisition time $T$ (with $A \propto T, C_{Y} \propto T$, thus $\boldsymbol{F} \propto T$ ). As a result, CNR is inversely proportional to $\sqrt{Y}$ and proportional to $\sqrt{T}$.

For the validation of Eqs. (21) and (22), we refer to [20], where repeated simulations were done using the post-smoothed MLEM reconstruction algorithm with a large number of iterations. For the pixel of interest $j$, the LLIR was obtained by reconstructing the noiseless projection data of the phantom with and without an impulse at $j$. The covariance image was calculated from the reconstructions of a large number of noisy projection data sets.

3) Extension to a ROI: Assuming local shift-invariance, it is valid to apply $Q_{L}^{j}$ and $Q_{C}^{j}$, which are derived for the particular pixel of interest $j$, to a small ROI centered at $j$ [17].

The vector of a uniform ROI can be written as

$$
R[i]= \begin{cases}0 & \text { if } i \notin \mathrm{ROI}, \\ 1 & \text { if } i \in \mathrm{ROI}\end{cases}
$$

where $i \in[1, \cdots, N]$ is the pixel index in the image space. Suppose the ROI is centered at pixel $j$, the mean in this ROI $\left(\mathrm{Mean}{ }^{\mathrm{ROI}}\right)$ and the variance on this mean $\left(\mathrm{VAR}^{\mathrm{ROI}}\right)$ can be approximated as

$$
\begin{aligned}
\operatorname{Mean}^{\text {ROI }}(\hat{\Lambda}) & \approx \frac{1}{N_{R}} R^{T} \boldsymbol{Q}_{L}^{j} R \\
\operatorname{VAR}^{\mathrm{ROI}}(\hat{\Lambda}) & \approx \frac{1}{N_{R}^{2}} R^{T} \boldsymbol{Q}_{C}^{j} R
\end{aligned}
$$

with $N_{R}$ the total number of pixels inside the ROI. The CNR in this ROI is

$$
\mathrm{CNR}^{\mathrm{ROI}}=\mathrm{Mean}^{\mathrm{ROI}} / \sqrt{\mathrm{VAR}^{\mathrm{ROI}}}
$$

\section{F. Collimator Aperture Optimization}

As we impose the target spatial resolution $\sigma_{t}$, the variance in the reconstruction is a function of the collimator aperture $\sigma_{c}$, because the variance is influenced by the geometric efficiency and the effect of collimator blurring, both of which are in turn determined by the collimator aperture. Therefore, it is possible to minimize the variance (or maximize the CNR) by a proper choice of the collimator aperture.

With the FBP-based calculation, the optimal value of the collimator aperture is derived by calculating the derivative of the denominator of the variance expression with respect to $\sigma_{c}$ (See Appendix-C and -D). This yields the minimal variance and the optimal collimator apertures $\sigma_{c}^{o p t}$.

With FIM, the optimal collimator aperture of a certain system is estimated as follows: 1) Choose a target resolution $\sigma_{t}$ and a particular pixel $j$ (or a ROI centered at $j$ ). 2) Calculate the CNR by Eqs. (21)-(24) (or Eqs. (26)-(28) for a ROI), using different collimator apertures ${ }^{3}$. 3) Plot the CNR curve as a function of the collimator aperture. 4) Interpolate the curve by the least-squares polynomial fitting method. 5) Locate the maximal CNR on the curve. 6) Define the collimator aperture which corresponds to the maximal CNR as the optimal collimator aperture $\sigma_{c}^{o p t}$ for the chosen target resolution. (This maximal CNR value is thus called optimized CNR in the remainder of the text.)

Using either the minimized variance (FBP) or the optimized CNR (FIM) of each collimator system as the input of Eq. (16) leads to an ultimate measure for the system comparison

$$
\operatorname{Gain}_{\mathrm{CNR}}^{\mathrm{opt}}=\frac{\mathrm{CNR}_{r}^{\mathrm{opt}}}{\mathrm{CNR}_{p}^{\mathrm{opt}}}=\sqrt{\frac{\mathrm{VAR}_{p}^{\text {min }}}{\mathrm{VAR}_{r}^{\text {min }}}}
$$

\section{NUMERICAL EXPERIMENTS}

In these experiments, synthetic noise-free data were computed for particular $\mathrm{PH}$ and $\mathrm{RS}$ system parameters using digitized versions of Eqs. (5)-(12), in order to compare the two systems.

We determined the optimal aperture for each collimator system, and then evaluated the relative performance of the $\mathrm{PH}$ and the RS collimators with their own optimal apertures. For the FBP-based method, the results were directly obtained from the analytical calculation. For the Fisher information-based method, we performed three sets of numerical experiments, which are described in this section. The system parameters used in these experiments are given in Table I. The acquisition time was 20 minutes for both the PH and the RS collimator systems.

\footnotetext{
${ }^{3}$ The collimator aperture $\sigma_{c}$ can be varied by changing $D, d_{c}$ or $h_{c}$, or their combinations (See Eq. (4)). In this study we fix $D$ and $d_{c}$ and only vary $h_{c}$.
} 
TABLE I

SYSTEM PARAMETERS

\begin{tabular}{|c|c|c|}
\hline Parameter & Planar imaging & Volume imaging \\
\hline Detector array & $97 \times 97$ & $97 \times 97$ \\
\hline Image dimension & $97 \times 97$ & $97 \times 97 \times 97$ \\
\hline Septa space \& Pixel size $\left(d_{c}\right)$ & $1.2 \mathrm{~mm}$ & $1.2 \mathrm{~mm}$ \\
\hline Detector width $(W)$ & $116.4 \mathrm{~mm}$ & $116.4 \mathrm{~mm}$ \\
\hline Projection angles $(\theta, \phi)$ & $\begin{array}{c}\text { PH: stationary } \\
\text { RS: } 64 \text { spin angles over } 2 \pi\end{array}$ & $\begin{array}{l}\text { PH: } 64 \text { rotation angles over } 2 \pi \\
\text { RS: } 64 \text { spin / } 64 \text { rotation angles over } 2 \pi\end{array}$ \\
\hline Raw data dimension & $\begin{array}{l}\text { PH: } 97 \times 97 \\
\text { RS: } 97 \times 64\end{array}$ & $\begin{array}{l}\text { PH: } 97 \times 97 \times 64 \\
\text { RS: } 97 \times 64 \times 64\end{array}$ \\
\hline
\end{tabular}

\section{A. Optimal Collimator Aperture}

First, the relation between the optimal collimator aperture $\left(\sigma_{p}^{o p t}, \sigma_{r}^{o p t}\right)$ and the target resolution $\sigma_{t}$ was derived for planar and volume imaging, respectively. The phantom used in planar imaging was a uniform disk with diameter $=54 \mathrm{~mm}$ and detector distance $D=100 \mathrm{~mm}$. The activity within the phantom was $\lambda=9.344 \mathrm{kBq} / \mathrm{cm}^{2}$. The optimal aperture was calculated only for the central point of the phantom.

The phantom used in volume imaging was a uniform sphere positioned at the center of the image space, with diameter $=$ $54 \mathrm{~mm}$ and $D=300 \mathrm{~mm}$. The activity of the phantom was $9.344 \mathrm{kBq} / \mathrm{cc}$. We assumed uniform attenuation within the phantom, with the attenuation coefficient $\mu=0.01505 \mathrm{~mm}^{-1}$. The optimal collimator aperture was individually calculated for the central point, 6 eccentric points and 2 ROIs. The eccentric points were selected equidistantly on the $\mathrm{X}$-axis or the $\mathrm{Z}$-axis (See Fig. 4(a)). The ROIs were spherical and located at the center of the phantom, with the same activity distribution as the phantom, and with diameter of $7.2 \mathrm{~mm}$ and $18.0 \mathrm{~mm}$, respectively.

\section{B. Optimized CNR Gain}

With the optimal collimator aperture derived in III-A, the optimized CNR gain (Gain ${ }_{\mathrm{CNR}}^{\mathrm{opt}}$ ) was calculated for the central point of the phantom, as a function of phantom diameter (diameter $=20.4-116.4 \mathrm{~mm})$ or detector distance $(D=$ $50-300 \mathrm{~mm}$ for planar imaging, $D=120-300 \mathrm{~mm}$ for volume imaging), with three different target resolutions $\left(\mathrm{FWHM}_{t}=9 \mathrm{~mm} / 10.8 \mathrm{~mm} / 12.6 \mathrm{~mm}\right)$. The phantom activity and the attenuation coefficient were the same as in III-A.

\section{Ellipsoid Phantom}

To verify the consistency of the acquisition models and reconstruction procedures, a post-smoothed MLEM reconstruction was performed for a simulated asymmetrical ellipsoidal phantom, with the $\mathrm{PH}$ and the RS collimator systems, respectively. As shown in Fig. 11(a), this phantom consists of a warm background $\left(\lambda=4.823 \mathrm{kBq} / \mathrm{cc}, \mu=0.0120 \mathrm{~mm}^{-1}\right)$, a hot spherical region $\left(\lambda=9.645 \mathrm{kBq} / \mathrm{cc}, \mu=0.0120 \mathrm{~mm}^{-1}\right)$ and a cold spherical region $\left(\lambda=2.411 \mathrm{kBq} / \mathrm{cc}, \mu=0.0045 \mathrm{~mm}^{-1}\right)$. The length of the semi-axes along/perpendicular to the axis of rotation were $36 \mathrm{~mm} / 27 \mathrm{~mm}$, respectively. The diameter of the two regions was $21.6 \mathrm{~mm}$. The distance between the center of the phantom and the detector was $80 \mathrm{~mm}$. The FWHM of the target resolution was $9 \mathrm{~mm}$.

First, we used FIM to optimize the collimator aperture for the central point of the phantom. Second, for each collimator system, the noiseless sinogram of this phantom with and without an impulse in its center were generated and reconstructed, yielding two reconstruction images. The LLIR was then obtained by subtracting these two reconstructions. Based on the shape of LLIR, the post-smoothing filter was defined by Eq. (23) and was applied to the reconstruction image.

In order to accelerate the convergence, ordered subset expectation maximization (OSEM) algorithm [21] was applied for the reconstruction. The iteration scheme for the $\mathrm{PH}$ system was $5 \times 32,5 \times 16,5 \times 8,5 \times 4,5 \times 2,5 \times 1$ (number of global iterations $\times$ number of subsets). For the RS system, the scheme of the subsets was the same and the number of iterations was doubled, because the reconstruction of RS data has a lower convergence speed than that of PH data [8].

\section{FBP: ANALYTICAL RESULTS}

\section{A. Optimal Collimator Aperture}

For the central point of a uniform symmetrical phantom, we obtain a linear relation between the target resolution $\sigma_{t}$ and the optimal collimator apertures $\sigma_{p}^{o p t}, \sigma_{r}^{o p t}$. For planar imaging

$$
\begin{aligned}
\sigma_{p}^{o p t} & =\sigma_{t} / \sqrt{2} \\
\sigma_{r}^{o p t} & =\sigma_{t} / 2
\end{aligned}
$$

and for volume imaging

$$
\begin{aligned}
& \sigma_{p}^{o p t}=\sigma_{t} / \sqrt{3} \\
& \sigma_{r}^{o p t}=\sigma_{t} / \sqrt{6}
\end{aligned}
$$

Note that for both imaging modes the simplified model leads to the remarkable relation

$$
\sigma_{p}^{o p t}=\sqrt{2} \sigma_{r}^{o p t}
$$




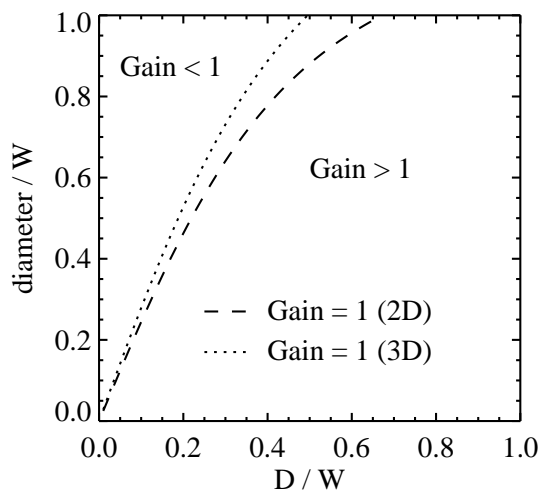

Fig. 2. Optimized CNR gain: influence of phantom size (diameter) and detector distance $D$ with respect to the detector width $W$. The upper left area is where Gain $_{\mathrm{CNR}}^{\mathrm{opt}}<1$, and the lower right area is where Gain ${ }_{\mathrm{CNR}}^{\mathrm{opt}}>1$.

\section{B. Optimized CNR Gain}

With the above mentioned optimal collimator apertures, the optimized CNR gain (RS versus $\mathrm{PH}$ ) are

$$
\begin{aligned}
\text { Gain }_{\mathrm{CNR}}^{\mathrm{opt}}(2 \mathrm{D}) & =1.116 \sqrt{\frac{\Omega_{W}}{\text { diameter }}} \\
\text { Gain }_{\mathrm{CNR}}^{\mathrm{opt}}(3 \mathrm{D}) & =1.192 \sqrt{\frac{\Omega_{W}}{\text { diameter }}}
\end{aligned}
$$

for planar (2D) and volume (3D) imaging, respectively. $\Omega_{W}$ is the effective detector width that is defined in Appendix-A, and diameter refers to the diameter of the phantom.

From Eqs. (35) and (36), it can be seen that Gain CNR $_{\mathrm{CN}}^{\mathrm{pt}}$ is independent of the target resolution $\sigma_{t}$, but depends on the size of the phantom (diameter) and the detector distance $(D)$ with respect to the detector width $(W)$. The influence of these factors on the gain is illustrated in Fig. 2 .

If $D / W>>1$, we have $\Omega_{W} \approx W$ (See Appendix-A). This implies that in the far-field case, RS is always better than $\mathrm{PH}$ as long as the phantom diameter is no larger than the detector width.

\section{FIM: NUMERICAL RESULTS}

\section{A. Optimal Collimator Aperture}

Fig. 3 shows the optimal collimator aperture of the central point of the 2D/3D symmetrical phantom, calculated by both proposed methods. The optimal apertures are plotted as a function of target resolution for both planar imaging (left) and volume imaging (right). For planar imaging, the results of the two methods are in good agreement. For volume imaging, there are small deviations for large target resolutions.

Fig. 4 shows the results for all points of interest calculated by FIM in volume imaging. Fig. 4(b) plots the optimal aperture for all the points at a target resolution of $\mathrm{FWHM}_{t}=9 \mathrm{~mm}$. The dashed lines plot the values calculated by Eqs. (32) and (33) as reference. From these plots we can see that the optimal aperture is insensitive to the location of the point. Fig. 4(c) shows the
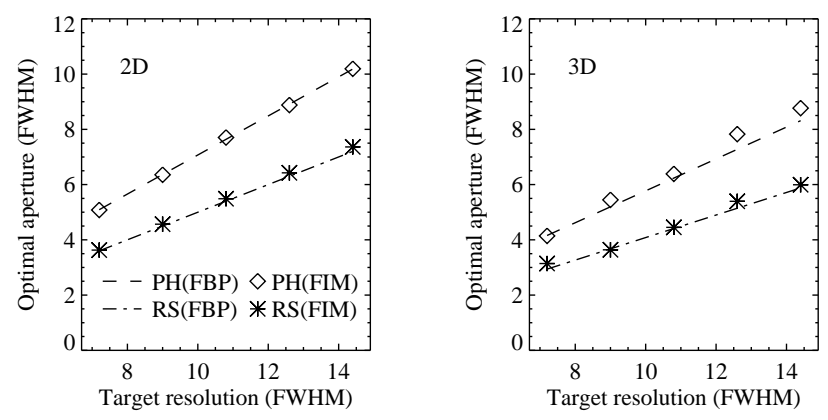

Fig. 3. Results of central point. The optimal collimator apertures calculated from the two methods are plotted as a function of the target resolution for planar imaging (left) and volume imaging (right), respectively. The unit of the FWHM is $\mathrm{mm}$.

CNRs of all points of interest, which were calculated using $\sigma_{p}^{o p t}$ and $\sigma_{r}^{o p t}$ of the central point of the phantom (point 0). For both $\mathrm{PH}$ and $\mathrm{RS}$, the CNR goes up when the point becomes more eccentric.

Fig. 5 shows the optimal aperture (left) and the ratio of the optimal apertures (right) of the central ROIs, calculated by FIM in volume imaging. From Fig. 5 we can see that: 1) The optimal aperture of the large ROI is always larger than that of the small ROI. 2) For the same target resolution, $\sigma_{p}^{o p t} \approx \sqrt{2} \sigma_{r}^{o p t}$.

\section{B. Optimized CNR Gain}

Fig. 6 and Fig. 7 show the optimized CNR gain at the central point of the phantom, plotted as a function of detector distance $D$ (left) or phantom diameter (right), calculated for planar and volume imaging, respectively. The target resolution is with $\mathrm{FWHM}_{t}=9 \mathrm{~mm}$. For planar imaging, we can see that the results of the two methods (FBP and FIM) are in good agreement for small phantoms or large $D$. For large phantoms or small $D$, the gains are slightly underestimated by the FBP-based method (Eq. (35)). For volume imaging on the other hand, the gains yielded by FIM are systematically smaller than those from FBP. Generally, the CNR gain increases with increased detector distance or smaller phantom size.

Fig. 8 plots the optimized CNR values that yielded the gain in Fig. 7. For volume imaging, the CNR acquired by either of the collimator systems increases with decreased detector distance or smaller phantom size.

Fig. 9 is similar as Fig. 7, with the optimized CNR gains calculated by FIM with different target resolutions. It confirms that CNR gain is not influenced by the choice of the target resolution, as long as the corresponding optimal aperture is used for each collimator system.

Fig. 10 shows the CNR gains of the central ROIs, calculated with the collimator apertures which minimize the variance in the central pixels. Very similar outcomes of the system comparison are yielded for the central pixel and the central ROIs. 


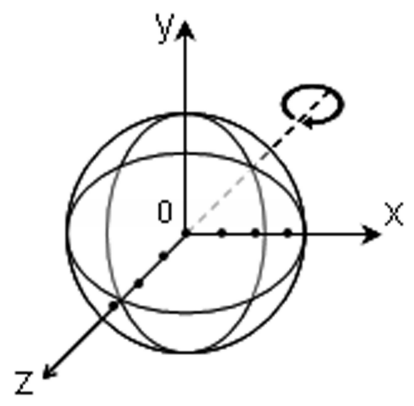

(a) Points of interest
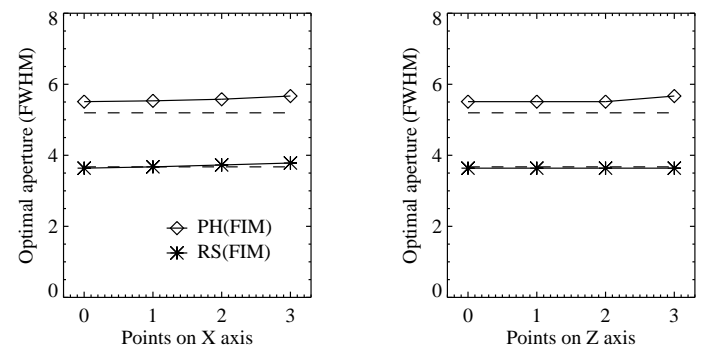

(b) Optimal aperture
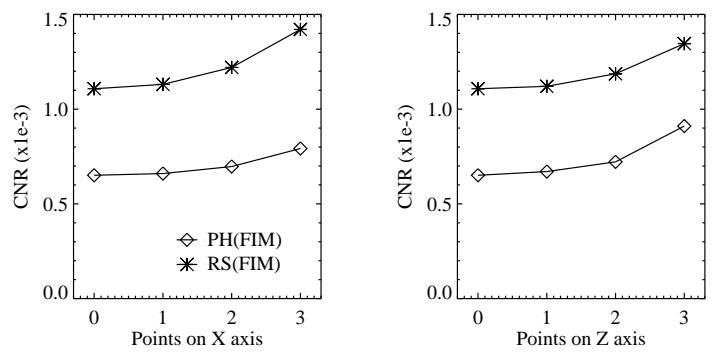

(c) CNR

Fig. 4. Results of eccentric points. (a) 3D spherical phantom and points of interest. $\mathrm{Z}$ is the axis of rotation, and $\mathrm{X}$ is perpendicular to $\mathrm{Z}$. (b) Optimal collimator aperture of all points of interest, calculated for the target resolution with $\mathrm{FWHM}_{t}=9 \mathrm{~mm}$. The dashed lines indicate the values obtained from the FBP calculation, which is only valid for point 0 . (c) CNR of all points of interest, calculated using the optimal apertures of point 0 , with $\mathrm{FWHM}_{t}=$ $9 \mathrm{~mm}$. The unit of the FWHM is $\mathrm{mm}$.
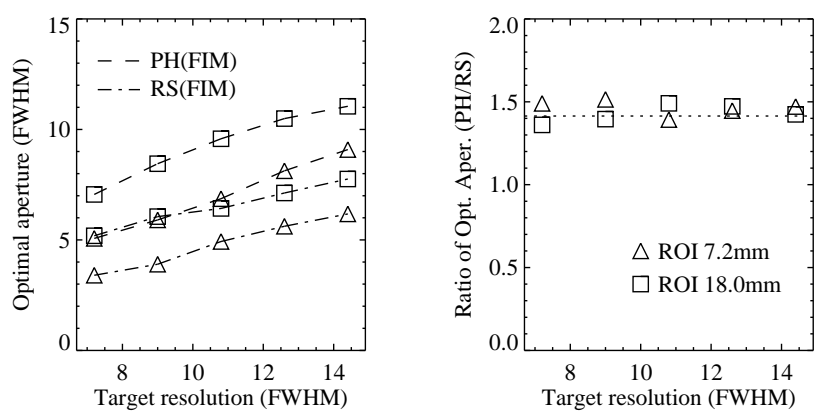

Fig. 5. Results of central ROIs. Left: Optimal aperture of the ROI as a function of the target resolution. Right: Ratio of the optimal aperture $\left(\sigma_{p}^{o p t} / \sigma_{r}^{o p t}\right)$. The dotted line indicates the value of $\sqrt{2}$. The unit of the FWHM is $\mathrm{mm}$.
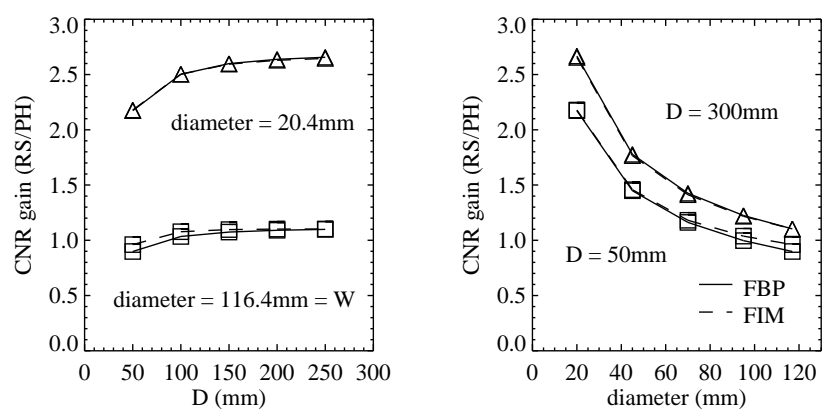

Fig. 6. Optimized CNR Gain in planar imaging with $\mathrm{FWHM}_{t}=9 \mathrm{~mm}$. Left: Gains plotted as a function of detector distance $D$ for two different phantom sizes (triangle: diameter $=20.4 \mathrm{~mm}$, square: diameter $=\mathrm{W}$ ). Right: Gains plotted as a function of phantom diameter at two different detector distances (triangle: $D=300 \mathrm{~mm}$, square: $D=50 \mathrm{~mm}$ ).
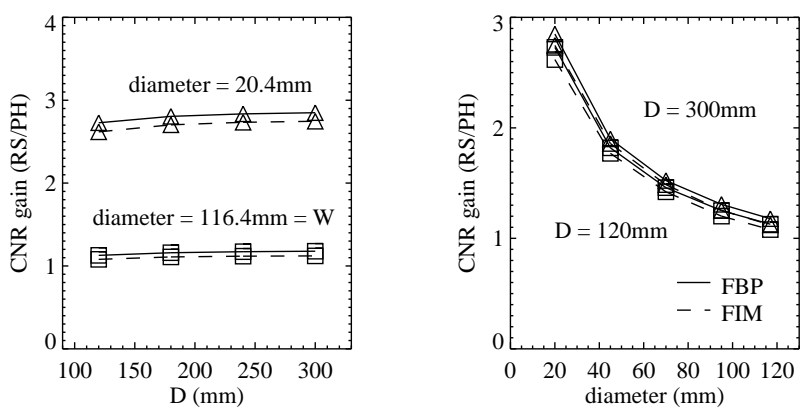

Fig. 7. Optimized CNR Gain in volume imaging with $\mathrm{FWHM}_{t}=9 \mathrm{~mm}$. Left: Gains plotted as a function of detector distance $D$ for two different phantom sizes (triangle: diameter $=20.4 \mathrm{~mm}$, square: diameter $=\mathrm{W}$ ). Right: Gains plotted as a function of phantom diameter at two different detector distances (triangle: $D=300 \mathrm{~mm}$, square: $D=120 \mathrm{~mm}$ ).
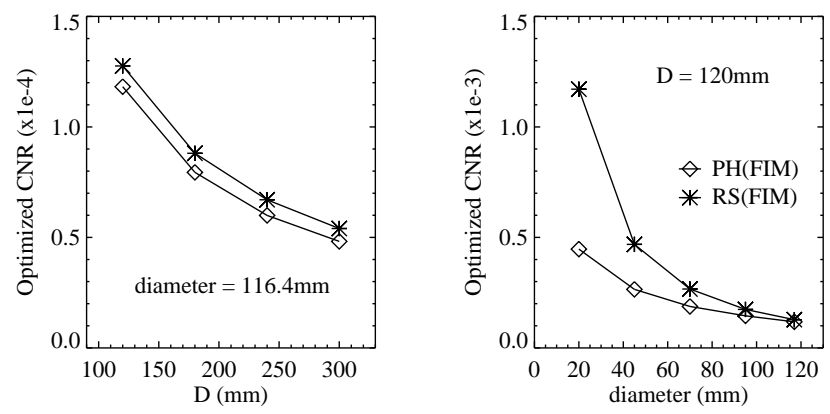

Fig. 8. Optimized CNR in volume imaging with $\mathrm{FWHM}_{t}=9 \mathrm{~mm}$. Left: CNR plotted as a function of detector distance $D$ for a phantom with diameter $=$ $W$. Right: CNR plotted as a function of phantom diameter with $D=120 \mathrm{~mm}$. 

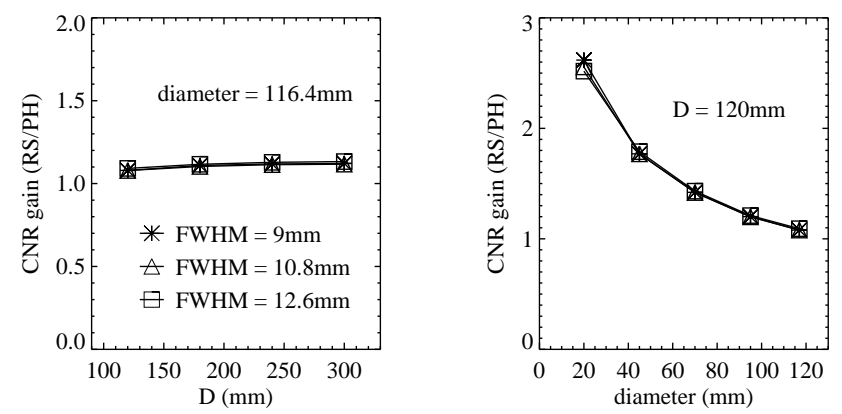

Fig. 9. Optimized CNR gain in volume imaging with different target resolutions $\left(\mathrm{FWHM}_{t}=9 \mathrm{~mm} / 10.8 \mathrm{~mm} / 12.6 \mathrm{~mm}\right)$. Left: Gains plotted as a function of detector distance $D$ for a phantom with diameter $=W$. Right: Gains plotted as a function of phantom diameter with $D=120 \mathrm{~mm}$.
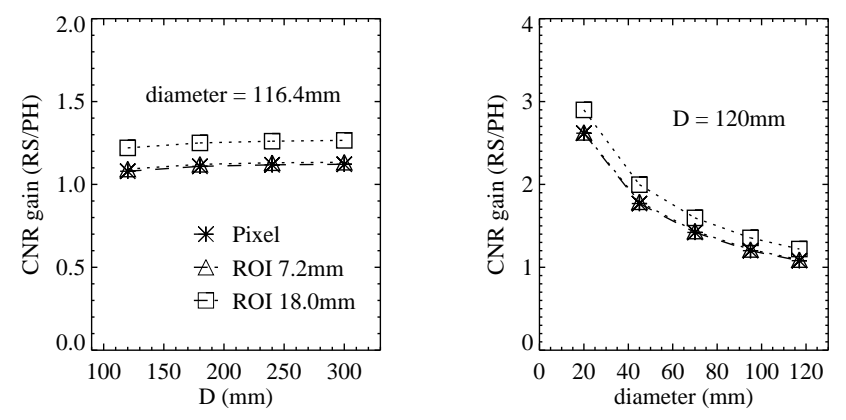

Fig. 10. CNR Gain of central ROIs in volume imaging with $\mathrm{FWHM}_{t}=9 \mathrm{~mm}$ The gains were calculated for the central ROIs with the optimal apertures minimizing the pixel variance. The asterisk represents the gain in the central pixel which is the same as the dashed line in Fig. 7. Left: Gains plotted as a function of detector distance $\mathrm{D}$ for a phantom with diameter $=W$. Right: Gains plotted as a function of phantom diameter with $D=120 \mathrm{~mm}$.

\section{Ellipsoid Phantom}

For the target resolution of $\mathrm{FWHM}_{t}=9 \mathrm{~mm}$, the optimal apertures for the central point of the ellipsoidal phantom are $\mathrm{FWHM}_{p}^{\text {opt }}=5.448 \mathrm{~mm}, \mathrm{FWHM}_{r}^{\text {opt }}=3.638 \mathrm{~mm}$ and $\mathrm{FWHM}_{p}^{o p t} / \mathrm{FWHM}_{r}^{o p t} \approx 1.497$. Fig. 11 shows the central sagittal slice of (a) the original phantom, (b) the phantom smoothed by the target resolution, (c)-(d) the PH and the RS MLEM reconstructions of the noiseless sinogram of this phantom, postsmoothed to the target resolution. The reconstructions look quite similar to the smoothed version of the original phantom.

\section{Discussion}

Previously, the validation of FIM has been performed for various multi-pinhole SPECT system configurations [16], as well as for time-of-flight positron emission tomography (TOFPET) [17]. As for the PH and the RS collimator system, we did the validation based on a $2 \mathrm{D}$ contrast phantom with postsmoothed MLEM reconstruction of 100 noise realizations [20]. As an example, the following result for the CNR in the ROIs in RS reconstruction was obtained in [20]. Plotting the CNR predicted by FIM with respect to the CNR calculated from the

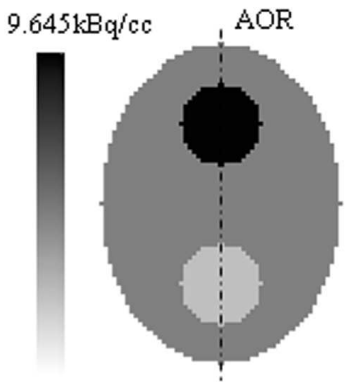

(a) Phantom

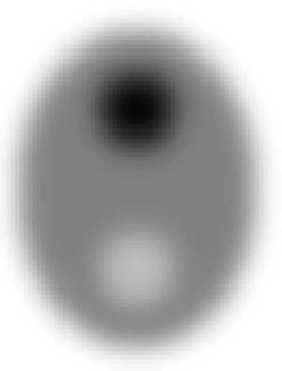

(c) Post-smoothed reconstruction ( $\mathrm{PH}$ )

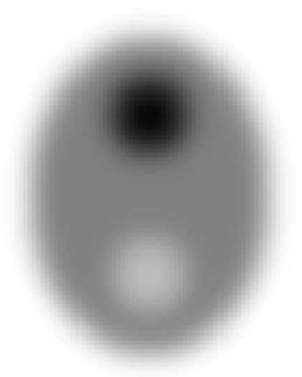

(b) Smoothed phantom

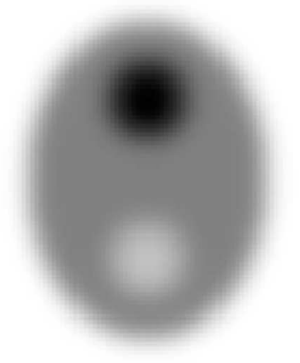

(d) Post-smoothed reconstruction (RS)
Fig. 11. Central sagittal slice of (a) ellipsoid phantom, (b) phantom smoothed with target resolution $\left(\mathrm{FWHM}_{t}=9 \mathrm{~mm}\right.$ ). (c)-(d) PH and RS MLEM reconstructions, calculated using their own optimal collimator apertures, postsmoothed to the target resolution. The dash dot line represents the axis of rotation.

reconstructions (See Fig. 2(a) in [20]), a slope of 1.04 can be derived using least squares fitting. In addition, $64.7 \%$ (resp. $94.1 \%$ ) of the plotted points are located in the $68.3 \%$ (resp. $95.4 \%$ ) confidence intervals (one and two standard deviations) centered on the identity line, indicating that the CNR estimated by FIM are in good accordance with those estimated from the repeated simulations. Details about this validation study can be found in [20]. Although the validation is only performed for planar imaging, we believe that FIM will be also valid for volume imaging due to its capability of accommodating shift-variant system response [16] and the attenuation within the phantom [17].

For the imaging systems studied here, FBP yields essentially the unweighted least squares (LS) solution. However, for a large symmetrical object, most of the projection values contributing to the reconstruction of a central point have the same expected value. Due to the assumed Poisson distribution, they also have the same variance, which means that LS can be approximately considered as weighted least squares (WLS). On the other hand, the approximate Fisher information-based method yields predictions that are in good agreement with reconstructions produced with MLEM or WLS followed by post-smoothing. Therefore, for the center of a large symmetrical object, postsmoothed LS, WLS and MLEM should all produce nearly the same variance at matched resolution. In our case, although the system models used for FBP and FIM are not identical due to the FBP-specific approximations, the difference is expected to have only minor influence on the variance of the central point of 
the object, where the modeling of the geometric efficiency and the collimator blurring is always accurate. Consequently, FBP calculation and FIM should agree well for the central points.

It can be seen from the results that the two methods indeed give very similar results for the central point of a uniform object. Although the FBP-based calculation involves some particular approximations, it provides insight into the mechanisms of noise propagation for the two types of collimators, and is expected to be accurate at least for the basic results (Eqs. (30)-(34)). FIM was validated for the proposed collimation geometries, and coincides well with the FBP-based method. In addition, FIM is more flexible in the sense that it can be applied to more realistic phantoms and more sophisticated system models, including the attenuation of the phantom and the depth-dependent collimator response in volume imaging, therefore it was used to extend and generalize the results of the FBP-based calculation.

The comparison was performed with several assumptions described in II-A. In fact, these assumptions (the septal penetration, the detector efficiency, etc.) are expected to have minor impact on the outcome of the collimator comparison. A nonnegligible septa thickness $t$ will introduce a scale factor of $d_{c}^{2} /\left(d_{c}+t\right)^{2}$ in $\sigma_{p}$ and of $d_{c} /\left(d_{c}+t\right)$ in $\sigma_{r}$. As a result, $\sigma_{c}^{o p t}$ in Eqs. (30)-(34) should also be scaled, and a scale factor of $\sqrt{\left(d_{c}+t\right) / d_{c}}$ should be applied to the optimized CNR gain in Eqs. (35)-(36). For a realistic acquisition system with intrinsic detector resolution $\sigma_{d} \neq 0, \sigma_{t}$ should be replaced by $\sqrt{\sigma_{t}^{2}-\sigma_{d}^{2}}$. With this replacement, our main results Eqs. (30)(36) will still hold for the central point of a symmetrical phantom.

From Eqs. (30)-(33), we found that the optimal collimator aperture is proportional to the target resolution that is imposed in the reconstruction. Similar results were presented in a study of pinhole imaging system [22], which claims that in order to minimize the variance of the emission-rate density estimate at a particular spatial resolution, the pinhole size should be proportional to that resolution. Furthermore, for a Gaussianshaped pinhole aperture, the FWHM of the pinhole aperture that yields the minimal variance in planar imaging equals the desired spatial resolution divided $\sqrt{2}$. The tradeoff between the spatial resolution and the noise with this Gaussian pinhole collimator is very similar to that with the $\mathrm{PH}$ collimator in our study. Therefore, Eqs. (30)-(33) could be also considered as a reference for the system optimization of other collimators whose configurations are similar to the proposed collimations in this paper.

Using the two analytical methods, it was also found that the optimal aperture of a line-integral acquisition system is always $\sqrt{2}$ times larger than that of a plane-integral acquisition system. This relation was first derived with the FBP-based calculation for a central point in an ideal phantom (Eq. (34)), and was then verified for eccentric points (Fig. 4) and central ROIs (Fig. 5) by FIM using more sophisticated system models. In addition, a similar ratio was also found for the central point of a non-symmetrical, non-uniform phantom (Fig. 11). Based on the results above, we propose to compare these two types of systems with their collimator aperture ratio equal to $\sqrt{2}$. The principle of using the optimal collimator aperture could be applied to any system comparison task at equal spatial resolution.

Our results show (Fig. 4(c)) that the eccentric pixels have a lower variance and higher CNR than central pixels in the phantom. This well-known observation [23] is explained by the fact that, with the $\mathrm{PH}(\mathrm{RS})$ system, some of the lines(planes) through eccentric pixels have a shorter intersection length (or less intersection area) with the phantom and are therefore less multiplexed with neighbouring pixels than the lines(planes) through the central pixels.

The CNR gain (Eqs. (35) and (36)) can also be written as a function of the geometric efficiency gain $F$ (with $F=E_{r} / E_{p}$ ). For example, for volume imaging, it yields

$$
\text { Gain }_{\mathrm{CNR}}^{\mathrm{opt}}=1.192 \sqrt{\frac{\Omega_{W}}{\text { diameter }}}=1.653 \sqrt{\frac{F \sigma_{t}}{\text { diameter }}}
$$

This expression is similar to those presented in [6], [7], where the formula was expressed as the gain in SNR rather than in CNR. Since we compared the two systems based on the same activity distribution, the SNR gain is actually equivalent to the CNR gain. The main differences between our approach and [6], [7] are that we compared PH and RS at a fixed target resolution, and that we used the optimized aperture for each collimator system.

The relative performance of the two systems for the central point of a uniform symmetrical phantom can be evaluated based on Figs. 2, 6 and 7. Fig. 2 explicitly gives the condition under which the RS will outperform $\mathrm{PH}$, and vice versa. Although the "Gain = 1" boundaries depicted in Fig. 2 are not accurate due to the FBP-specific approximations, they can be considered as references for the collimator comparison when combined with Figs. 6 and 7. It can be seen that the comparative result is ultimately determined by the object size and the objectto-detector distance $D$. Generally, the optimized CNR gain increases with smaller object size (which is in agreement with [5]-[8]) or with larger object-to-detector distance. One should note that for tomographic scans the absolute CNR value actually decreases as the detector distance increases, therefore the object should be always positioned as close as possible to the collimator, regardless of the system in use.

In this paper, we mainly focused on uniform symmetrical phantoms, while realistic tracer distributions in human and preclinical imaging are usually very non-uniform. To verify the consistency between the projector and back-projector, we also present the reconstruction images of a non-uniform, nonsymmetrical ellipsoid phantom using the optimal aperture of its central point calculated by FIM. With the modeling of depthdependent blurring and the attenuation, the reconstruction in volume imaging is very time-consuming. Therefore we did not consider numerical verification of FIM in this setting.

Only objects smaller than the field of view (FOV) of the collimators were considered in this study to avoid the possibly confounding effects of the data truncation problem which is 
still poorly understood. When an object is larger than the FOV in transaxial direction, both $\mathrm{PH}$ and RS systems may suffer from increased variance and artifacts, depending on the amount of data truncation. For axial truncation, however, $\mathrm{PH}$ has the advantage because the axial out-of-FOV activity remains undetected and does not create artifacts. In contrast, the RS system will detect a contribution from this activity. This contribution will increase the variance in the reconstruction. Moreover, unless the acquisition can be well modeled as plane integrals, artifact-free reconstruction may be problematic as well. However, only considering objects fitting in the FOV, we already found that for very large objects, the $\mathrm{PH}$ collimator should be recommended rather than the RS collimator. Consequently, considering even larger objects which will cause additional problems for RS, will not change that recommendation.

In this study, the CNR gains for two different ROI sizes were quantified with the collimator aperture optimized for the pixel variance. Strictly speaking, for optimal quantification of an object of interest, both the collimator aperture and the target resolution should be adjusted to match the size of that object. However, in practice, we usually produce a single image with a fixed aperture size, applying a certain amount of smoothing, and then use that image for all kinds of tasks such as visual inspection, definition of ROIs with different sizes and quantification of the ROI-values. In this sense, it is reasonable to perform the comparative task for a single pixel or the ROIs with different sizes based on the same system parameters. The ranking for the relative system performance is found to be very similar regardless of the object of interest.

\section{CONCLUSION}

In this study, we compare the $\mathrm{PH}$ and the RS collimators using the optimal collimator aperture that yields the minimal pixel/ROI variance at a prescribed spatial resolution. Two analytical methods, the FBP-based calculation and the Fisher information-based method, are applied to compute the optimized CNR using a simplified collimator blurring model and a realistic depth-dependent blurring model, respectively. It is shown that the two methods yield very similar results for the central point in a uniform disk or sphere phantom. In contrast to the FBP-based calculation, FIM can predict the LLIR and variance of any point of interest, or even region of interest in arbitrary non-uniform phantoms.

It is found that the variance in the reconstruction image varies with the collimator aperture, and that the optimal collimator aperture is proportional to the target spatial resolution imposed in the reconstruction. For equal spatial resolution in the reconstruction, RS always needs more post-smoothing than $\mathrm{PH}$. Therefore, the optimal collimator aperture of a RS system is always smaller than that of a $\mathrm{PH}$ system. Our results suggest that a fair comparison between the performance of the two types of collimators can be achieved using a ratio of $\sqrt{2}$ between the $\mathrm{PH}$ and the RS collimator apertures. With the optimal apertures, the expressions of the CNR gain (RS versus $\mathrm{PH}$ ) are derived in different imaging modes. Pixel variance and ROI variances yield a very similar ranking for the relative system performance, which is determined by both the object size and the object-todetector distance.

\section{ACKNOWLEDGMENT}

We wish to thank Roel Van Holen (ELIS, Ghent University) for many useful discussions about the rotating slat collimator systems.

\section{APPENDICES}

\section{A. Effective Detector Width}

We calculate the effective detector width for the RS collimator (see Eqs. (35) and (36)). We denote $(\alpha, \gamma)$ the coordinates of a point on a plane centered between two adjacent slat septa, with $\alpha$ measured along the axis lying in the detector plane along the slat septa, and $\gamma$ measured along the axis orthogonal to the detector. The coordinate center is taken at the center of the detector strip, so that $\gamma$ is the distance between the point and the detector surface. For this point, the effective detector width $\Omega_{W}(\alpha, \gamma)$ is defined as

$$
\Omega_{W}(\alpha, \gamma)=\int_{-W / 2}^{+W / 2} \cos ^{3} \beta(\alpha-l, \gamma) d l
$$

where $W$ is the width of the detector along the slat septa, and $\beta(\alpha-l, \gamma)$ is the angle between the normal to the detector and the trajectory of the photon (emitted from point $(\alpha, \gamma)$ and detected at location $l$ along the slat septa):

$$
\cos \beta(\alpha-l, \gamma)=\frac{\gamma}{\sqrt{(\alpha-l)^{2}+\gamma^{2}}}
$$

With the FBP-based method, we focus on the central point of the image space, for which $(\alpha, \gamma)=(0, D)$. For convenience, we use $\Omega_{W}$ (without the arguments $\alpha$ and $\gamma$ ) to denote the effective detector width for that central point. Eq. (A-1) yields then

$$
\Omega_{W}=\int_{-W / 2}^{+W / 2} \cos ^{3} \beta(l, D) d l=\frac{D W}{\sqrt{D^{2}+W^{2} / 4}}
$$

If $D>>W$, Eq. (A-3) becomes

$$
\Omega_{W} \approx W
$$

\section{B. Geometric Efficiency}

We calculate the geometric efficiencies $E_{p}$ and $E_{r}$ used in the data acquisition models of Eqs. (5), (6), (9) and (11) for the PH and the RS collimator systems, respectively.

For the $\mathrm{PH}$ collimator, the geometric efficiency $E_{p}$ is shiftinvariant. For square holes, $E_{p}$ is estimated as [18]

$$
E_{p}=\frac{d_{p}^{2}}{4 \pi h_{p}^{2}}=\frac{\mathrm{FWHM}_{p}^{2}}{4 \pi D^{2}}=\frac{2 \ln 2 \sigma_{p}^{2}}{\pi D^{2}}
$$

where $d_{p}$ and $h_{p}$ are the length and the height of the holes, respectively, and $\sigma_{p}$ (or $\mathrm{FWHM}_{p}$ ) represents the $\mathrm{PH}$ collimator aperture that corresponds to the center of the image space. 
For the RS collimator, the geometric efficiency $E_{r}$ is shiftvariant. The geometric efficiency of a small detector element $d l$ at location $l$ along the slat for a point source located at $(\alpha, \gamma)$ in the plane defined by two adjacent slat septa is [4]

$$
E_{r}(\alpha-l, \gamma) d l=\frac{d_{r} d l}{4 \pi \gamma h_{r}} \cos ^{3} \beta(\alpha-l, \gamma)
$$

where $d_{r}$ is the distance between two adjacent slat septa, $h_{r}$ is the height of the septa, and $\beta$ is defined in Eq. (A-2). Eq. (B-2) is used to defined the system matrix $A$ for the FIM method, where the detector is modeled as an array of individual square detector elements of length $d l=d_{r}$.

For the FBP-based calculation, the position-dependence of $E_{r}$ is ignored and a single value is used for the entire image space. This value is calculated as the geometric efficiency of the central point of the image space, integrated over the whole detector strip. We denote this value as $E_{r}$, without arguments. Substituting $(\alpha, \gamma)=(0, D)$ in Eq. (B-2) and integrating over the length of the detector strip, we obtain

$$
E_{r}=\int_{-W / 2}^{+W / 2} E_{r}(l, D) d l=\frac{d_{r}}{4 \pi D h_{r}} \Omega_{W}
$$

with $\Omega_{W}$ given by Eq. (A-3). Using Eqs. (2) and (4), this can also be written as

$$
E_{r}=\frac{\mathrm{FWHM}_{r}}{4 \pi D^{2}} \Omega_{W}=\frac{\sqrt{2 \ln 2} \sigma_{r}}{2 \pi D^{2}} \Omega_{W}
$$

where $\sigma_{r}$ (or $\mathrm{FWHM}_{r}$ ) is the RS collimator aperture corresponding to the center of the image space.

\section{FBP Calculation for Planar Imaging}

In this appendix, we derive the optimal collimator aperture (Eqs. (30) and (31)) and the optimized CNR gain (Eq. (35)) for the two collimator systems by simplifying the acquisition models (Eqs. (5) and (6)) for planar imaging.

For a $2 \mathrm{D}$ tracer distribution $\lambda(\vec{x})$ in a plane parallel to the detector surface, we model the $\mathrm{PH}$ data $\left(y_{p}\right)$ as a convolution between $\lambda(\vec{x})$ and a 2D Gaussian PSF with standard deviation $\sigma_{p}$. The RS data $\left(y_{r}\right)$ is modeled as a convolution between the ideal 2D Radon transform of $\lambda(\vec{x})$ and a 1D Gaussian PSF with standard deviation $\sigma_{r}$. The data are scaled by the geometric efficiency $E_{c}$ and the acquisition time $T_{c}$. Replacing the subscript $c$ with $p$ and $r$, the acquired data of the PH and the RS systems are written as

$$
\begin{aligned}
y_{p}(\vec{x}) & =T_{p} \times E_{p} \times\left(\lambda(\vec{x}) \otimes G_{2}\left(\vec{x}, \sigma_{p}\right)\right) \\
y_{r}(s, \phi) & =T_{r} \times E_{r} \times\left(y_{r 0}(s, \phi) \otimes G_{1}\left(s, \sigma_{r}\right)\right)
\end{aligned}
$$

where $s$ is the distance between the slat interval and the spin axis on the gamma camera, and $\phi \in[0, \pi]$ denotes the angular position of the RS collimator as it spins on the fixed camera. $\otimes$ represents the convolution operation, and $G_{i}$ denotes the $i$ thdimensional isotropic Gaussian function

$$
G_{i}\left(x_{1}, x_{2}, \cdots, x_{i}, \sigma\right)=\frac{1}{(\sqrt{2 \pi} \sigma)^{i}} e^{-\left(x_{1}^{2}+x_{2}^{2}+\cdots+x_{i}^{2}\right) / 2 \sigma^{2}}
$$

Assume the same acquisition time, we have

$$
T_{r}=T_{p} / \pi
$$

since RS spins over $\pi$ during the acquisition. $E_{p}$ and $E_{r}$ are defined in Eq. (B-1) and Eq. (B-4), respectively.

In Eq. (C-2), $y_{r 0}$ is the ideal unblurred 2D Radon transform of $\lambda(\vec{x})$

$$
y_{r 0}(s, \phi)=\int_{\mathbb{R}^{2}} d \vec{x} \lambda(\vec{x}) \delta\left(\vec{x} \cdot \vec{u}_{\phi}-s\right)
$$

with $\vec{u}_{\phi}=(-\sin \phi, \cos \phi)$ the unit vector orthogonal to the slats in the detector plane.

We assume the noise on the data is Poisson noise. Hence, the covariance in the data is

$$
\begin{gathered}
\operatorname{Cov}\left(y_{p}(\vec{x}), y_{p}(\vec{y})\right)=y_{p}(\vec{x}) \delta(\vec{x}-\vec{y}) \\
\operatorname{Cov}\left(y_{r}(s, \phi), y_{r}\left(s^{\prime}, \phi^{\prime}\right)\right)=y_{r}(s, \phi) \delta\left(s-s^{\prime}\right) \delta\left(\phi-\phi^{\prime}\right)
\end{gathered}
$$

We aim to do the reconstructions with a fixed target resolution $\sigma_{t}$, with the constraints of $\sigma_{t}>\sigma_{p}$ and $\sigma_{t}>\sigma_{r}$. To reconstruct the image for $\mathrm{PH}$, we apply a Gaussian filter with standard deviation of $\sqrt{\sigma_{t}^{2}-\sigma_{p}^{2}}$ to yield the target resolution $\sigma_{t}$ in the reconstructed image. For RS, we process the data in the same way as for $\mathrm{PH}$, and then do the filtered-backprojection. The data processing is done in the Fourier domain. The reconstruction images $\lambda_{t p}(\vec{x}), \lambda_{t r}(\vec{x})$ in the spatial domain can be described as

$$
\begin{aligned}
& \lambda_{t p}(\vec{x})=\frac{1}{T_{p} E_{p}} \times\left(y_{p}(\vec{x}) \otimes G_{2}\left(\vec{x}, \sqrt{\sigma_{t}^{2}-\sigma_{p}^{2}}\right)\right) \\
& \lambda_{t r}(\vec{x})=\frac{1}{T_{r} E_{r}} \int_{0}^{\pi} d \phi\left[y_{r}(s, \phi) \otimes h_{r}(s)\right]_{s=\vec{x} \cdot \vec{u}_{\phi}}
\end{aligned}
$$

with

$$
h_{r}(s)=\int_{\mathbb{R}} d \nu|\nu| e^{-2 \pi^{2}\left(\sigma_{t}^{2}-\sigma_{r}^{2}\right) \nu^{2}} e^{+2 \pi i \nu s}
$$

where $v$ is the frequency conjugate to the sinogram variable $s$. The variance of the $\mathrm{PH}$ estimate $\lambda_{t p}(\vec{x})$ is

$$
\begin{aligned}
\operatorname{VAR}\left(\lambda_{t p}(\vec{x})\right) & =\frac{1}{\left(T_{p} E_{p}\right)^{2}} \int_{\mathbb{R}^{2}} d \vec{y} \int_{\mathbb{R}^{2}} d \vec{z} G_{2}\left(\vec{y}, \sqrt{\sigma_{t}^{2}-\sigma_{p}^{2}}\right) \\
& \times G_{2}\left(\vec{z}, \sqrt{\sigma_{t}^{2}-\sigma_{p}^{2}}\right) \operatorname{Cov}\left(y_{p}(\vec{x}-\vec{y}), y_{p}(\vec{x}-\vec{z})\right) \\
& =\frac{1}{\left(T_{p} E_{p}\right)^{2}}\left(G_{2}\left(\vec{x}, \sqrt{\sigma_{t}^{2}-\sigma_{p}^{2}}\right)\right)^{2} \otimes y_{p}(\vec{x})(\mathrm{C}-10)
\end{aligned}
$$

The variance of the RS estimate $\lambda_{t r}(\vec{x})$ is

$$
\begin{aligned}
& \operatorname{VAR}\left(\lambda_{t r}(\vec{x})\right)=\frac{1}{\left(T_{r} E_{r}\right)^{2}} \int_{0}^{\pi} d \phi \int_{\mathbb{R}} d s \int_{0}^{\pi} d \phi^{\prime} \int_{\mathbb{R}} d s^{\prime} h_{r}(s) h_{r}\left(s^{\prime}\right) \\
& \times \operatorname{Cov}\left(y_{r}\left(\vec{x} \cdot \vec{u}_{r}(\phi)-s, \phi\right), y_{r}\left(\vec{x} \cdot \vec{u}_{r}\left(\phi^{\prime}\right)-s^{\prime}, \phi^{\prime}\right)\right) \\
& =\frac{1}{\left(T_{r} E_{r}\right)^{2}} \int_{0}^{\pi} d \phi\left[y_{r}(s, \phi) \otimes\left|h_{r}(s)\right|^{2}\right]_{s=\vec{x} \cdot \vec{u}_{\phi}}(\mathrm{C}-11)
\end{aligned}
$$

The convolution kernels $\left(G_{2}\left(\vec{x}, \sqrt{\sigma_{t}^{2}-\sigma_{p}^{2}}\right)\right)^{2}$ and $\left|h_{r}(s)\right|^{2}$ in Eq. (C-10) and Eq. (C-11) are sharply peaked around the origin, therefore we use the data acquired by the central detector to 
approximate the data acquired by all the other detectors [24]. Assuming the activity in $\lambda(\vec{x})$ is $\lambda / s e c$, we have

$$
\begin{aligned}
y_{p}(\vec{x}) & \approx \lambda \times T_{p} \times E_{p} \\
y_{r}(s, \phi) & \approx \lambda \times T_{r} \times E_{r} \times \text { diameter }
\end{aligned}
$$

We have the variance of the central point

$$
\begin{aligned}
\left.\operatorname{VAR}\left(\lambda_{t p}(\vec{x})\right)\right|_{\vec{x}=0} & \approx \frac{\lambda}{T_{p} E_{p}} \times \frac{1}{4 \pi\left(\sigma_{t}^{2}-\sigma_{p}^{2}\right)} \\
& =\frac{D^{2} \lambda}{8 \ln 2 T \sigma_{p}^{2}\left(\sigma_{t}^{2}-\sigma_{p}^{2}\right)} \\
\left.\operatorname{VAR}\left(\lambda_{t r}(\vec{x})\right)\right|_{\vec{x}=0} & \approx \frac{\lambda \times \text { diameter }}{T_{r} E_{r}} \times \frac{\pi^{-3 / 2}}{16\left(\sigma_{t}^{2}-\sigma_{r}^{2}\right)^{3 / 2}} \\
& =\frac{\sqrt{\pi} D^{2} \lambda \times \text { diameter }}{8 \sqrt{2 \ln 2} \Omega_{W} T \sigma_{r}\left(\sigma_{t}^{2}-\sigma_{r}^{2}\right)^{3 / 2}}(\mathrm{C}-
\end{aligned}
$$

We can find the minimal variance by deriving the denominator in Eqs. (C-14) and (C-15) with respect to $\sigma_{p}$ and $\sigma_{r}$, respectively. The optimal collimator aperture thus is

$$
\begin{aligned}
& \sigma_{p}^{o p t}=\sigma_{t} / \sqrt{2} \\
& \sigma_{r}^{o p t}=\sigma_{t} / 2
\end{aligned}
$$

With these optimal apertures, the minimal variance of the central point in the reconstruction yields

$$
\begin{aligned}
\operatorname{VAR}_{p}^{\text {min }} & =\frac{D^{2} \lambda}{2 \ln 2 T \sigma_{t}^{4}} \\
\mathrm{VAR}_{r}^{\text {min }} & =\frac{2 \sqrt{\pi} D^{2} \lambda \times \text { diameter }}{3 \sqrt{6 \ln 2} \Omega_{W} T \sigma_{t}^{4}}
\end{aligned}
$$

The optimized gain for the central point is

$$
\begin{aligned}
\text { Gain }^{\text {opt }} & =\sqrt{\frac{\mathrm{VAR}_{p}^{\text {min }}}{\mathrm{VAR}_{r}^{\text {min }}}} \\
& =\frac{3^{3 / 4}}{(8 \ln 2 \times \pi)^{1 / 4}} \times \sqrt{\frac{\Omega_{W}}{\text { diameter }}} \\
& =1.116 \sqrt{\frac{\Omega_{W}}{\text { diameter }}}
\end{aligned}
$$

\section{FBP Calculation for Volume Imaging}

In this appendix, we derive the optimal collimator aperture (Eqs. (32) and (33)) and the optimized CNR gain (Eq. (36)) by simplifying the acquisition models (Eqs. (9) and (11)) for volume imaging.

For volume imaging, the collimator and the gamma camera rotate around the axis of rotation $\overrightarrow{l_{z}}$ to obtain complete tomographic data. For $\mathrm{PH}$, the data is denoted as $y_{p}(v, \theta, z)$ where $v$ and $\theta$ are the usual sinogram coordinates in that slice, and $z$ is the axial coordinate of the slice. For RS, the acquired data is parametrized as $y_{r}(s, \phi, \theta)$, where $s$ is the distance between the slat and the collimator spinning axis, $\phi$ is the rotational position of the collimator as it spins on the fixed camera, and $\theta$ is the rotational position of the camera around the $z$ axis.

$$
\begin{aligned}
& y_{p}(v, \theta, z)=T_{p} \times E_{p} \times\left(y_{p 0}(v, \theta, z) \otimes G_{2}\left(v, z, \sigma_{p}\right)\right) \\
& y_{r}(s, \phi, \theta)=T_{r} \times E_{r} \times\left(y_{r 0}(s, \phi, \theta) \otimes G_{1}\left(s, \sigma_{r}\right)\right)
\end{aligned}
$$

With $\theta \in[0,2 \pi]$ and $\phi \in[0, \pi]$, the acquisition time become

$$
\begin{aligned}
& T_{p}=T / 2 \pi \\
& T_{r}=T / 2 \pi^{2}
\end{aligned}
$$

$y_{p 0}(v, \theta, z)$ denotes the unblurred 2D Radon transform of $\lambda(\vec{x})=\lambda(x, y, z)$ in slice $z$

$$
y_{p 0}(v, \theta, z)=\int_{\mathbb{R}^{3}} d \vec{x} \lambda(\vec{x}) \delta\left(\vec{x} \cdot \vec{l}_{z}-z\right) \delta\left(\vec{x} \cdot \vec{u}_{\theta}-v\right)
$$

with

$$
\vec{u}_{\theta}=(-\sin \theta, \cos \theta, 0)
$$

$y_{r 0}(s, \phi, \theta)$ denotes the ideal 3D Radon transform of $\lambda(\vec{x})$ over the plane $\Pi\left(\vec{u}_{\phi}, s\right)$ that is located at a distance $s$ from the origin of the $3 \mathrm{D}$ coordinate system and orthogonal to a unit vector $\vec{u}_{\phi}$

$$
y_{r 0}(s, \phi, \theta)=\int_{\mathbb{R}^{3}} d \vec{x} \lambda(\vec{x}) \delta\left(\vec{x} \cdot \vec{u}_{\phi}-s\right)
$$

with

$$
\vec{u}_{\phi}=(-\sin \phi \cos \theta,-\sin \phi \sin \theta, \cos \phi)
$$

The reconstruction images are [19]

$$
\begin{aligned}
& \lambda_{t p}(\vec{x})=\frac{1}{T_{p} E_{p}} \int_{0}^{2 \pi} d \theta\left[y_{p}(v, \theta, z) \otimes h_{p}(v, z)\right]_{s=\vec{x} \cdot \vec{u}_{\theta}} \\
& \lambda_{t r}(\vec{x})=\frac{1}{2 T_{r} E_{r}} \int_{0}^{2 \pi} d \theta \int_{-1}^{1} d \cos \phi\left[y_{r}(s, \phi, \theta) \otimes h_{r}(s)\right]_{s=\vec{x} \cdot \vec{u}}(\mathrm{D}-10
\end{aligned}
$$

with

$$
\begin{aligned}
h_{p}(\nu, z) & =\int_{\mathbb{R}} d \nu \int_{\mathbb{R}} d \nu_{z}|\nu| e^{-2 \pi^{2}\left(\sigma_{t}^{2}-\sigma_{p}^{2}\right)\left(\nu^{2}+\nu_{z}^{2}\right)} e^{+2 \pi i\left(\nu+\nu_{z} z\right)}(\mathrm{D}-11) \\
h_{r}(s) & =\int_{\mathbb{R}} d \nu \nu^{2} e^{-2 \pi^{2}\left(\sigma_{t}^{2}-\sigma_{r}^{2}\right) \nu^{2}} e^{+2 \pi i \nu s}
\end{aligned}
$$

For the variance calculation, similar approximations as in Eqs. (C-12) and (C-13) are made for the data

$$
\begin{aligned}
& y_{p}(s, \theta, z) \approx \lambda \times T_{p} \times E_{p} \times \text { diameter } \\
& y_{r}(s, \phi, \theta) \approx \lambda \times T_{r} \times E_{r} \times \pi \times(\text { diameter } / 2)^{2}
\end{aligned}
$$

Following the same thoughts as for planar imaging, we can derive the expressions for the variance of the central point in the reconstructions

$$
\begin{aligned}
& \left.\operatorname{VAR}\left(\lambda_{t p}(\vec{x})\right)\right|_{\vec{x}=0}=\frac{D^{2} \lambda \times \text { diameter }}{64 \ln 2 T \sigma_{p}^{2}\left(\sigma_{t}^{2}-\sigma_{p}^{2}\right)^{2}} \\
& \left.\operatorname{VAR}\left(\lambda_{t r}(\vec{x})\right)\right|_{\vec{x}=0}=\frac{3 \pi^{3 / 2} D^{2} \lambda \times \text { diameter }^{2}}{256 \sqrt{8 \ln 2} \Omega_{W} T \sigma_{r}\left(\sigma_{t}^{2}-\sigma_{r}^{2}\right)^{5 / 2}}
\end{aligned}
$$

The optimal collimator aperture can be easily calculated from Eqs. (D-15) and (D-16)

$$
\begin{aligned}
& \sigma_{p}^{o p t}=\sigma_{t} / \sqrt{3} \\
& \sigma_{r}^{o p t}=\sigma_{t} / \sqrt{6}
\end{aligned}
$$

Hence, the minimal variance of the central point in the reconstruction is

$$
\begin{aligned}
& \mathrm{VAR}_{p}^{\text {min }}=\frac{27 D^{2} \lambda \times \text { diameter }}{256 \ln 2 \sigma_{t}^{6}} \\
& \mathrm{VAR}_{r}^{\text {min }}=\frac{81 \pi^{3 / 2} D^{2} \lambda \times \text { diameter }}{2} \\
& 1600 \sqrt{10 \ln 2} \Omega_{W} T \sigma_{t}^{6}
\end{aligned}
$$


The optimized gain for the central point is

$$
\begin{aligned}
\text { Gain }^{\text {opt }} & =\sqrt{\frac{\mathrm{VAR}_{p}^{\text {min }}}{\mathrm{VAR}_{r}^{\text {min }}}} \\
& =\frac{5^{5 / 4}}{3^{1 / 2} \pi^{3 / 4}(8 \ln 2)^{1 / 4}} \times \sqrt{\frac{\Omega_{W}}{\text { diameter }}} \\
& =1.192 \sqrt{\frac{\Omega_{W}}{\text { diameter }}}
\end{aligned}
$$

\section{REFERENCES}

[1] D. Gagnon, G. L. Zeng, J. M. Links, J. J. Griesmer, and F. C. Valentino, "Design considerations for a new solid-state gamma-camera SOLSTICE.' IEEE Nuclear Science Symposium Conference Record, pp. 1156-1160, 2002.

[2] S. Webb, M. A. Flower and R. J. Ott, "Geometric efficiency of a rotating slit-collimator for improved planar gamma-camera imaging." Phys. Med. Biol., 38, pp. 627-638, 1993.

[3] S. Webb, D. M. Binnie, M. A. Flower and R. J. Ott, "Monte Carlo modelling of the performance of a rotating slit-collimator for improved planar gamma camera imaging." Phys. Med. Biol., 37(5), pp. 1095-1108, 1992.

[4] S. Vandenberghe, R. Van Holen, S. Staelens and I. Lemahieu, "System characteristics of SPECT with a slat collimated strip detector." Phys. Med. Biol., 51, pp. 391-405, 2006.

[5] M. A. Lodge, D. M. Binnie, M. A. Flower and S. Webb, "The experimental evaluation of a prototype rotating slat collimator for planar gamma camera imaging." Phy. Med. Biol., 40, pp. 427-448, 1995.

[6] M. A. Lodge, S. Webb, M. A. Flower and D. M. Binnie, "A prototype rotating slat collimator for single photon emission computed tomography." IEEE Trans. Med. Imag., 15(4), pp. 500-511, 1996.

[7] B. Zhang and G. L. Zeng, "Study of noise propagation and the effects of insufficient numbers of projection angles and detector samplings for iterative reconstruction using planar-integral data." Med. Phys., 33(9), pp. 3124-3134, 2006.

[8] R. Van Holen, S. Vandenberghe, S. Staelens and I. Lemahieu, "Comparing planar image quality of rotating slat and parallel hole collimation: influence of system modeling." Phys. Med. Biol., 53, pp. 1989-2002, 2008.

[9] R. Van Holen, S. Vandenberghe, S. Staelens and I. Lemahieu, "Comparison of 3D SPECT imaging with a rotating slat collimator and a parallel hole collimator." IEEE Nuclear Science Symposium Conference Record, pp. 4592-4597, 2008.

[10] W. Wang, W. Hawkins and D. Gagnon, "3D RBI-EM reconstruction with spherically-symmetric basis function for SPECT rotating slat collimator.' Phys. Med. Biol., 49, pp. 2273- 2292, 2004.

[11] H. H. Barrett, J. L. Denny, R. F. Wagner and K. J. Myers, ”Objective assessment of image quanlity. II. Fisher information, Fourier crosstalk, and figures of merit for task performance." J. Opt. Soc. Am. A, 12(5), pp. 834-852, 1995.

[12] K. Vunckx, J. Nuyts, B. Vanbilloen, M. De Saint-Hubert, D. Vanderghinste, D. Rattat, F. M. Mottaghy, and M. Defrise, "Optimized multipinhole design for mouse imaging." IEEE Trans. Nucl. Sci., vol. 56(5), pp. 26962705, 2009.

[13] J. A. Fessler, "Mean and variance of implicitly defined biased estimators (such as penalized maximum likelihood): applications to tomography." IEEE Trans. Image Processing, 5(3), pp. 493-506, 1996.

[14] J. A. Fessler and W. L. Rogers, "Spatial resolution properties of penalized likelihood image reconstruction: space-invariant tomographs." IEEE Trans. Image Proc., 5(9), pp. 1346-1358, 1996.

[15] J. Qi and R. M. Leahy, "A theoretical study of the contrast recovery and variance of MAP reconstructions from PET data." IEEE Trans. Med. Imag., 18(4), pp. 293-305, 1999.

[16] K. Vunckx, D. Bequé, M. Defrise and J. Nuyts, ”Single and multipinhole collimator design evaluation method for small animal SPECT." IEEE Trans. Med. Imag., 27(1), pp. 36-46, 2008.

[17] K. Vunckx, L. Zhou, S. Matej, M. Defrise and J. Nuyts, "Fisher information-based evaluation of image quality for time-of-flight PET." IEEE Trans. Med. Imag., 29(2), pp. 311-321, 2010.

[18] H. Wieczorek and A. Goedicke, "Analytical model for SPECT detector concepts." IEEE Trans. Nuc. Sci., 53(3), pp. 1102-1112, 2006.
[19] H. H. Barrett, "Foundation of imaging science." Wiley Interscience, 2003.

[20] L. Zhou, K. Vunckx and J. Nuyts, "Comparison between parallel hole and rotating slat collimation with a contrast phantom using an analytical method." IEEE Nuclear Science Symposium Conference Record, pp. 3678-3681, 2009.

[21] H. M. Hudson and R. S. Larkin, "Accelerated image reconstruction using ordered subsets of projection data." IEEE Trans. Med. Imag., 13(4), pp. 601-609, 1994

[22] J. A. Fessler, "Spatial resolution and noise tradeoffs in pinhole imaging system design: a density estimation approach." Opt. Express, 2(6), pp. 237-253, 1998.

[23] N. M. Alpert, D. A. Chesler, J. A. Correia, R. H. Ackerman, J. Y. Chang, S. Finklestein, S. M. Davis, G. L. Brownell and J. M. Taveras, "Estimation of the local statistical noise in emission computed tomography." IEEE Trans. Med. Imag., MI-1(2), pp. 142-146, 1982.

[24] M. Defrise, D. W. Townsend and F. Deconinck, "Statistical noise in threedimensional positron tomography." Phys. Med. Biol., 35(1), pp. 131-138, 1990. 